\title{
UPPER CRITICAL FIELD AND LOCATION OF SURFACE NUCLEATION OF SUPERCONDUCTIVITY
}

\author{
CHAMP SURCRITIQUE ET LOCALISATION \\ DE LA NUCLÉATION DE SURFACE \\ EN SUPRACONDUCTIVITÉ
}

\author{
Bernard HELFFER $^{\mathrm{a}, *}$, Xing-Bin PAN ${ }^{\mathrm{b}, \mathrm{c}}$ \\ ${ }^{a}$ Département de mathématiques, UMR CNRS 8628, bat. 425, Université Paris-Sud, \\ 91405 Orsay cedex, France \\ ${ }^{\mathrm{b}}$ Department of Mathematics, National University of Singapore, Singapore 119260 \\ c Department of Mathematics, Zhejiang University, Hangzhou 310027, People's Republic of China
}

Received 20 February 2001, revised 27 July 2001

ABSTRACT. - In this paper we improve the estimate obtained by Lu-Pan on the value of the upper critical field $H_{C_{3}}(\kappa)$ for a cylindrical superconductor with cross section $\Omega$ being an arbitrary 2-dimensional smooth bounded domain. We also show that, when a homogeneous magnetic field is applied along the axis of the cylinder with magnitude of the field close to $H_{C_{3}}$, superconductivity nucleates first at the surface of the sample where the curvature of $\partial \Omega$ is maximal.

(C) 2003 L'Association Publications de l'Institut Henri Poincaré. Published by Elsevier B.V. All rights reserved

MSC: 35Q55; 82D55

Keywords: Ginzburg-Landau system; Superconductivity; Nucleation; Upper critical field; Eigenvalues; Schrödinger operator with magnetic fields

RÉSUMÉ. - Nous améliorons dans cet article l'estimation obtenue par Lu-Pan de la varleur du champ surcritique $H_{C_{3}}(\kappa)$ pour un supraconducteur cylindrinque dont la section $\Omega$ est un ouvert régulier borné dans le plan.

Nous montrons aussi que, lorsqu'un champ magnétique constant parallèle à l'axe du cylindre est appliqué avec une intensité voisine de $H_{C_{3}}(\kappa)$, la supraconductivité apparaît d'abord aux points du bord où la courbure $\partial \Omega$ est maximale.

(C) 2003 L'Association Publications de l'Institut Henri Poincaré. Published by Elsevier B.V. All rights reserved

\footnotetext{
* Corresponding author.

E-mail addresses: bernard.helffer@math.u-psud.fr (B. Helffer), matpanxb@nus.edu.sg (X.-B. Pan).
} 


\section{Introduction}

Let us consider a cylindrical superconducting sample of type 2 with cross section $\Omega$ being an arbitrary bounded smooth domain in $\mathbb{R}^{2}$, and place the sample in an applied magnetic field. It is well known that, if the applied field is very strong then the sample loses superconducting property. As the field is gradually reduced to a certain value $H_{C_{3}}$ called the upper critical field, the nucleation of superconductivity occurs, see $[8,23,24]$.

We are interested in the estimate of the value of $H_{C_{3}}(\kappa)$ when the Ginzburg-Landau parameter $\kappa$ is large, and in the localization of the nucleation of superconductivity. These problems have been studied by many physicists, see Saint-James and De Gennes [22], Saint-James and Sarma [23], and Tinkham [24]. More recently, a lot of papers devoted to the mathematical analysis on these problems have appeared. Among them we mention the works of Chapman [5] and Bernoff and Sternberg [3] based on some formal analysis, Bauman, Philips and Tang [2] for the rigorous analysis on disks, Giorgi and Phillips [12], Lu and Pan [16-21] and del Pino, Felmer and Sternberg [9] for rigorous analysis on general domains. Our main concern is the effect of the domain geometry on the value of $H_{C_{3}}$ and on the location of superconductivity nucleation.

Before stating our main results we recall that, for a cylindrical superconductor of infinite height with cross section $\Omega$ and subject to an applied magnetic field along the cylindrical axis, the behavior of superconductivity can be described by a (global) minimizer $(\psi, \mathcal{A})$ of the Ginzburg-Landau functional

$$
\mathcal{G}(\psi, \mathcal{A})=\int_{\Omega}\left\{\left|\nabla_{\kappa \mathcal{A}} \psi\right|^{2}+\kappa^{2}|\operatorname{curl} \mathcal{A}-\mathcal{H}|^{2}+\frac{\kappa^{2}}{2}\left(|\psi|^{2}-1\right)^{2}\right\} \mathrm{d} x .
$$

The Euler equation is the 2-dimensional Ginzburg-Landau system (see $[6,8,10,11,23]$ )

$$
\begin{cases}-(\nabla-i \kappa \mathcal{A})^{2} \psi=\kappa^{2}\left(1-|\psi|^{2}\right) \psi, & \\ \operatorname{curl}^{2} \mathcal{A}=-\frac{i}{2 \kappa}(\bar{\psi} \nabla \psi-\psi \nabla \bar{\psi})-|\psi|^{2} \mathcal{A}+\operatorname{curl} \mathcal{H}, & \text { in } \Omega, \\ \frac{\partial \psi}{\partial v}-i \kappa \mathcal{A} \psi \cdot v=0, & \text { on } \partial \Omega .\end{cases}
$$

Here $\psi$ is a complex-valued function called order parameter, $\mathcal{A}$ is a real vector field called magnetic potential, $\mathcal{H}$ is the applied magnetic field, $i=\sqrt{-1}, \kappa$ is the GinzburgLandau parameter given by the ratio of the London penetration depth and the coherence length of the superconductor, $v$ is the unit out-normal vector at the boundary of $\Omega$. Here we use the notations

$$
\begin{aligned}
& \partial_{j}=\frac{\partial}{\partial x_{j}}, \quad \nabla_{\mathcal{A}} \psi=\nabla \psi-i \psi \mathcal{A}, \\
& \nabla_{\mathcal{A}}^{2} \psi=(\nabla-i \mathcal{A})^{2} \psi=\Delta \psi-i[2 \mathcal{A} \cdot \nabla \psi+\psi \operatorname{div} \mathcal{A}]-|\mathcal{A}|^{2} \psi, \\
& \operatorname{curl} \mathcal{A}=\partial_{1} \mathcal{A}_{2}-\partial_{2} \mathcal{A}_{1}, \\
& \operatorname{curl}^{2} \mathcal{A}=\left(\partial_{2}(\operatorname{curl} \mathcal{A}),-\partial_{1}(\operatorname{curl} \mathcal{A})\right) .
\end{aligned}
$$

Note that in (1.1) the unit of length is the penetration depth. Also note that in [1621] a more general boundary condition introduced by de Gennes was considered. For 
simplicity we consider the homogeneous Neumann boundary condition. However, the ideas and methods used in this paper apply as well for this boundary condition.

Notations. - In the following, a (global) minimizer $(\psi, \mathcal{A})$ of the Ginzburg-Landau functional is called a minimal solution of the Ginzburg-Landau system (1.1). We call a complex-valued function $\psi$ an order parameter if there exists a real vector field $\mathcal{A}$ such that $(\psi, \mathcal{A})$ is a minimal solution of the Ginzburg-Landau system (1.1).

Throughout this paper we assume

$$
\mathcal{H}(x) \equiv \sigma \mathbf{e}_{3},
$$

where $\sigma$ is a constant. We shall treat $\sigma$ as a parameter and consider the behavior of minimizers as $\sigma \rightarrow \infty$. It is well known that there exists a unique smooth vector field $\mathbf{F}$ on $\bar{\Omega}$ such that

$$
\operatorname{curl} \mathbf{F}=1 \quad \text { and } \quad \operatorname{div} \mathbf{F}=0 \quad \text { in } \Omega, \quad \mathbf{F} \cdot v=0 \quad \text { on } \partial \Omega .
$$

Note that, under the assumption, $(0, \sigma \mathbf{F})$ is a trivial critical point of the functional $\mathcal{G}$. Moreover, $(0, \sigma \mathbf{F})$ is the only minimizer if $\sigma$ is large enough, which means that a sufficiently strong applied magnetic field penetrates the entire superconductor and completely destroys the superconductivity (see [19], or Theorem A below).

A mathematical definition for $H_{C_{3}}$ was given in [19]:

$$
H_{C_{3}}(\kappa)=\inf \{\sigma>0:(0, \sigma \mathbf{F}) \text { is the only minimizer of } \mathcal{G}\} .
$$

THEOREM A [19]. - For any bounded 2-dimensional simply-connected domain $\Omega$ of class $C^{3}$ we have:

(1) $\lim _{\kappa \rightarrow \infty} H_{C_{3}}(\kappa) / \kappa=1 / \beta_{0}$, where $\beta_{0}$ is the lowest eigenvalue of (2.6).

(2) If the homogeneous applied field is sufficiently close to $H_{C_{3}}$, superconductivity nucleates at the surface of the sample.

Conclusion (2) in Theorem A is called surface nucleation. The precise meaning is the following. Assume that the applied field $\mathcal{H}$ is given in (1.2), with the magnitude $\sigma$ satisfying

$$
(1+\mathrm{o}(1)) \frac{\kappa}{\beta_{0}} \leqslant \sigma<H_{C_{3}}(\kappa) .
$$

Then, for any $\delta>0$, there exists $\kappa(\delta)>0$ such that, for all $\kappa>\kappa(\delta)$, the order parameters $\psi$ must satisfy

$$
\|\psi\|_{L^{\infty}(\Omega)}<\delta .
$$

For any $x \in \Omega$ and any $\eta>0$, there exists $\kappa(x, \eta)>0$ such that, for all $\kappa>\kappa(x, \eta)$,

$$
\frac{|\psi(x)|}{\|\psi\|_{L^{\infty}(\Omega)}}<\eta
$$

Moreover, the order parameters exhibit a boundary layer in the neighborhood of $\partial \Omega$ within distance $\mathrm{O}(1 / \kappa)$ to $\partial \Omega$. For more details see [19]. 
However, the information given in (2) does not give the optimal localization of the surface nucleation. It turns out that the localization depends on the gap between the applied field and $H_{C_{3}}$. In fact, it was shown in [19] that if the gap between the applied field and $H_{C_{3}}$ is not very small, one can observe superconductivity uniformly along the entire surface of the sample. For a more precise statement, see [19] (Section 5). On the other hand, if the gap is small, the lower bound estimates on $H_{C_{3}}$ (which are related to the upper bound estimates of the lowest eigenvalue of Schrödinger operator with magnetic field) given in [3] and [19] suggest that superconductivity can only be observed near the maximum points of the curvature of $\partial \Omega$.

In this paper, based on the very recent work by Helffer and Morame [15] on the asymptotical behavior of the lowest eigenvalue, we shall improve the estimate of $H_{C_{3}}$ given in [19], and show that, under a homogeneous magnetic field $\mathcal{H}=H \mathbf{e}_{3}$ parallel to the axis direction $\mathbf{e}_{3}$ of the cylinder with magnitude close to $H_{C_{3}}$, superconductivity nucleates first at the points in the surface of the sample where the curvature of $\partial \Omega$ is maximal.

Throughout this paper $\Omega$ is a smooth simply-connected bounded domain in $\mathbb{R}^{2}$. Let $\kappa_{r}(x)$ be the curvature function of $\partial \Omega$, and let us introduce

$$
\begin{aligned}
& \kappa_{\max }=\max _{x \in \partial \Omega} \kappa_{r}(x), \quad \kappa_{\min }=\min _{x \in \partial \Omega} \kappa_{r}(x), \\
& \mathcal{N}(\partial \Omega)=\left\{x \in \partial \Omega: \kappa_{r}(x)=\kappa_{\max }\right\} .
\end{aligned}
$$

Denote by $s=s(x)$ the arclength of $\partial \Omega$, denote by $x(s)$ a point at $\partial \Omega$, and write the curvature function $\kappa_{r}(x(s))$ by $\kappa_{r}(s)$. We say that a maximum point $x\left(s_{0}\right)$ of the curvature is non-degenerate, if $\kappa_{r}^{\prime \prime}\left(s_{0}\right) \neq 0$.

For a $C^{1}$ domain $\Omega$, there is a geometric constant $\varepsilon(\Omega)>0$ such that the distance function to the boundary is regular in the domain $\{x \in \bar{\Omega}$ : $\operatorname{dist}(x, \partial \Omega)<\varepsilon(\Omega)\}$, and for any $0<\delta<\varepsilon(\Omega)$, we can introduce a new coordinates $(s, t)$ in a neighborhood of $\partial \Omega$

$$
\Omega_{\delta}=\{x \in \bar{\Omega}: \operatorname{dist}(x, \partial \Omega)<\delta\}
$$

to straighten the boundary $\partial \Omega$, where $s=s(x)$ measures the arclength and $t=t(x)=$ $\operatorname{dist}(x, \partial \Omega)$ measures the normal distance to the boundary, see [15] (Appendix B). Let us fix $\delta_{0}<\varepsilon(\Omega)$. We shall identify $x(s, 0)$ with $x(s)$. We can measure the tangential distance from the point $x(s)$ to $\mathcal{N}(\partial \Omega)$ along $\partial \Omega$, and we shall denote this distance by $d_{t}(x(s), \mathcal{N}(\partial \Omega))$. Then we define the tangential distance from $x=x(s, t)$ to $\mathcal{N}(\partial \Omega)$ by

$$
d_{t}(x(s, t), \mathcal{N}(\partial \Omega)) \equiv d_{t}(x(s), \mathcal{N}(\partial \Omega)) .
$$

To every point $x=x(s, t) \in \Omega_{\delta_{0}}$, we can assign a unique point $x(s)=x(s, 0) \in \partial \Omega$. Hence, we can define

$$
\kappa_{r}(x)=\kappa_{r}(x(s)) \quad \text { for } x=x(s, t) \in \Omega_{\delta} .
$$

As we shall see later that, order parameters concentrate at a tubular neighborhood of $\partial \Omega$ and exponentially decay in the normal direction away from $\partial \Omega$. We shall derive various integral estimates for order parameters in the tubular neighborhood, and the curvature 
function will play an important role in these estimates. Therefore, in the following we extend the function $\kappa_{r}(x)$ onto $\Omega$ such that it is continuous on $\bar{\Omega}$, equal to $\kappa_{r}(x(s))$ for $x=x(s, t) \in \Omega_{\delta_{0}}$, and equal to a positive constant in $\Omega \backslash \Omega_{2 \delta_{0}}$. Then $\kappa_{r}(x)$ is well-defined on $\bar{\Omega}$. When $\Omega$ is of class $C^{4}$ we also require $\kappa_{r}(x)$ to be of class $C^{2}$.

Our main results in this paper are Theorems 1.1, 1.2 and 1.3. Theorem 1.1 gives an estimate of $H_{C_{3}}(\kappa)$ up to the second term. Theorems 1.2 and 1.3 give a quantitative version of the property that superconductivity nucleates at the maximum points of the curvature as the applied magnetic field decreases from $H_{C_{3}}$, and the condensation behavior of the order parameters depends on the gap $H_{C_{3}}-\sigma$ between the applied field $\sigma$ and the upper critical field $H_{C_{3}}$.

THEOREM 1.1 (Asymptotics of $H_{C_{3}}$ ). - Assume that $\Omega$ is a bounded simply-connected 2-dimensional domain of class $C^{4}$. There exists a universal constant $C_{1}>0$ such that, for $\kappa$ large, we have

$$
H_{C_{3}}(\kappa)=\frac{\kappa}{\beta_{0}}+\frac{C_{1}}{\beta_{0}^{3 / 2}} \kappa_{\max }+\mathrm{O}\left(\kappa^{-1 / 3}\right) .
$$

THEOREM 1.2 (Location of nucleation: general domains). - Let $\Omega$ be as in Theorem 1.1 and let

$$
\rho=\frac{\beta_{0}^{3 / 2}}{C_{1}}\left[H_{C_{3}}(\kappa)-\sigma\right] .
$$

The order parameters $\psi$ have the following concentration behaviors.

(1) Assume that $0<\rho=\mathrm{o}(1)$ as $\kappa \rightarrow \infty$. Then there exist positive constants $a_{0}, \ell_{0}$, $\kappa_{0}$ and $M_{0}$ such that, for all $\kappa>\kappa_{0}$, we have

$$
\int_{\Omega}|\psi|^{2} \exp \left(a_{0} \sqrt{\kappa}\left[\kappa_{\max }-\kappa_{r}(x)\right]\right) \mathrm{d} x \leqslant M_{0} \exp \left(\ell_{0} \rho \sqrt{\kappa}\right) \int_{\Omega}|\psi|^{2} \mathrm{~d} x .
$$

(2) Assume that $\varepsilon_{0}<\rho<\kappa_{\max }-\kappa_{\min }$, where $\varepsilon_{0}>0$. Then there exist positive constants $a, M$ and $\kappa_{0}^{\prime}$ such that, for all $\kappa>\kappa_{0}^{\prime}$ we have ${ }^{1}$

$$
\int_{\Omega}|\psi|^{2} \exp \left(a \sqrt{\kappa}\left[\kappa_{\max }-\kappa_{r}(x)-\rho\right]_{+}^{3 / 2}\right) \mathrm{d} x \leqslant M \kappa^{1 / 3} \int_{\Omega}|\psi|^{2} \mathrm{~d} x .
$$

THEOREM 1.3 (Location of nucleation: non-degenerate domains). - Assume that $\Omega$ is a bounded simply-connected 2-dimensional domain of class $C^{4}$ and $\mathcal{N}(\partial \Omega)$ consists of non-degenerate maximum points of curvature. The order parameters $\psi$ have the following concentration behaviors.

(1) Assume that

$$
0<H_{C_{3}}(\kappa)-\sigma \leqslant L_{1} \kappa^{-1 / 3}
$$

for some constant $L_{1}>0$. Then there exist positive constants $a_{1}, r_{1}, \ell_{1}, B_{1}$ and $\kappa_{1}$ such that, for all $\kappa>\kappa_{1}$, we have

\footnotetext{
${ }^{1}$ We use the notation $a_{+}=\max \{a, 0\}$.
} 


$$
\begin{aligned}
& \int_{\bar{\Omega} \backslash\left\{x \in \bar{\Omega}: \operatorname{dist}(x, \partial \Omega)<r_{1} \kappa^{-5 / 6}, d_{t}(x, \mathcal{N}(\partial \Omega))<\ell_{1} \kappa^{-1 / 6}\right\}}|\psi|^{2} \mathrm{~d} x \\
& \quad \leqslant B_{1} \exp \left(-a_{1} \kappa^{1 / 6}\right)\|\psi\|_{L^{2}(\Omega)}^{2} .
\end{aligned}
$$

(2) Assume that: ${ }^{2} L_{2} \kappa^{-1 / 3} \ll H_{C_{3}}-\sigma=\mathrm{o}(1)$ for some constant $L_{2}>0$. Then there exist positive constants $a_{2}, r_{2}, \ell_{2}, \kappa_{2}$ and $B_{2}$ such that, for all $\kappa>\kappa_{2}$, we have

$$
\begin{aligned}
& \left.\int_{\bar{\Omega} \backslash\left\{x \in \bar{\Omega}: \operatorname{dist}(x, \partial \Omega)<r_{2} \rho \kappa^{-1 / 2},\right.} \mid \psi d_{t}(x, \mathcal{N}(\partial \Omega))<\ell_{2} \sqrt{\rho}\right\} \\
& \leqslant B_{2} \exp \left(-a_{2} \rho \sqrt{\kappa}\right)\|\psi\|_{L^{2}(\Omega)}^{2},
\end{aligned}
$$

here $\rho=\frac{\beta_{0}^{3 / 2}}{C_{1}}\left[H_{C_{3}}(\kappa)-\sigma\right]$.

Remark 1.4. - We will see in Proposition 4.2 that, if the applied field is close to but below $H_{C_{3}}$, the order parameters $\psi$ decay exponentially in the normal direction and have a boundary layer of the order $\mathrm{O}\left(\kappa^{-1}\right)$. Thus superconductivity nucleates in a boundary layer with thickness of order $\mathrm{O}\left(\kappa^{-1}\right)$. Theorems 1.2 and 1.3 describe in a weak sense the exponential decay of the order parameters in the tangential direction within the boundary layer, and hence describe the way in which superconductivity nucleates and expands as the gap $H_{C_{3}}(\kappa)-\sigma$ between the applied field and $H_{C_{3}}$ increases. In the case where all the maximum points of the curvature $\kappa_{r}(s)$ are non-degenerate, we have the following conclusions:

(1) If the gap is of order $\mathrm{O}\left(\kappa^{-1 / 3}\right)$, then superconductivity nucleates near the set $\mathcal{N}(\partial \Omega)$ of the maximum points of the boundary curvature, with a normal distance of order $\mathrm{O}\left(\kappa^{-5 / 6}\right)$ and with a tangential distance of order $\mathrm{O}\left(\kappa^{-1 / 6}\right)$ to $\mathcal{N}(\partial \Omega)$.

(2) If the gap tends to zero but is still much larger than the order $\mathrm{O}\left(\kappa^{-1 / 3}\right)$, then superconductivity still nucleates near the maximum points of the boundary curvature. But now it is located within a neighborhood of $\mathcal{N}(\partial \Omega)$ with a normal distance $\mathrm{O}\left(\kappa^{-1 / 2}\left(H_{C_{3}}-\sigma\right)\right)$ and with a tangential distance of order $\left(H_{C_{3}}-\sigma\right)^{1 / 2}$.

(3) If the gap does not tend to zero but remains bounded below by $C_{1} \beta_{0}^{-3 / 2} \rho$, where $0<\rho<\kappa_{\max }-\kappa_{\min }$, then superconductivity is localized inside a neighborhood of $\mathcal{N}_{\rho}(\partial \Omega)$, a subset of the surface

$$
\mathcal{N}_{\rho}(\partial \Omega) \equiv\left\{x(s) \in \partial \Omega: \kappa_{r}(s) \geqslant \kappa_{\max }-\rho\right\} .
$$

Moreover, if $\kappa_{r}^{\prime}(s) \neq 0$ whenever $\kappa_{r}(s)=\kappa_{\max }-\rho$, then superconductivity is located inside a neighborhood of $\mathcal{N}_{\rho}(\partial \Omega)$ with thickness $\mathrm{O}\left(\kappa^{-1 / 3}\right)$. In fact, under this condition, there exists a constant $c$ (which depends on $\rho$ ) such that,

$$
\kappa_{\max }-\kappa_{r}(s)-\rho \geqslant c d_{t}\left(x(s), \mathcal{N}_{\rho}(\partial \Omega)\right) \quad \text { for all } x(s) \in \partial \Omega \backslash \mathcal{N}_{\rho}(\partial \Omega) .
$$

\footnotetext{
${ }^{2}$ Our convention is that, for two positive quantities depending on a parameter $\kappa$, we will write $a \ll b$ if $a / b=\mathrm{o}(1)$ as $\kappa \rightarrow \infty$.
} 
Since the order parameters $\psi$ decay exponentially in the normal direction, for any $0<\delta<1 / 3$, we use (1.7) to find that

$$
\int_{\Omega \cap\left\{\operatorname{dist}\left(x, \mathcal{N}_{\rho}(\partial \Omega)\right) \geqslant \kappa^{-\delta}\right\}}|\psi|^{2} \mathrm{~d} x \leqslant C \kappa^{1 / 3} \exp \left(-a_{3} \kappa^{(1-3 \delta) / 2}\right) \int_{\Omega}|\psi|^{2} \mathrm{~d} x,
$$

where $C>0$ is independent of $\kappa$, and $a_{3}=a c^{3 / 2}$.

(4) We believe that, if the gap is greater than $\frac{C_{1}}{\beta_{0}^{3 / 2}}\left(\kappa_{\max }-\kappa_{\min }\right)$, namely, if

$$
\sigma<\frac{\kappa}{\beta_{0}}+\frac{C_{1}}{\beta_{0}^{3 / 2}} \kappa_{\min }
$$

then order parameters concentrate uniformly along the entire boundary. We should mention that uniform concentration along boundary was proved in [19] under a stronger condition: $\frac{1}{\beta_{0}} \kappa^{2 / 3}-\sigma \kappa^{-1 / 3} \rightarrow+\infty$ as $\kappa \rightarrow+\infty$. This condition means that: $\sigma \ll \frac{\kappa}{\beta_{0}}-L \kappa^{1 / 3}$ for some $L>0$.

Remark 1.5. - We believe that the error term in (1.5) is $\mathrm{O}\left(\kappa^{-1 / 2}\right)$. In fact, using Proposition 2.3, we have, for some positive constants $m_{1}$ and $m_{2}$,

$$
\frac{\kappa}{\beta_{0}}+\frac{C_{1}}{\beta_{0}^{3 / 2}} \kappa_{\max }-m_{1} \kappa^{-1 / 2}<H_{C_{3}}(\kappa)<\frac{\kappa}{\beta_{0}}+\frac{C_{1}}{\beta_{0}^{3 / 2}} \kappa_{\max }+m_{2} \kappa^{-1 / 3} .
$$

Remark 1.6. - The estimates given in Theorems 1.2 and 1.3 are not optimal.

(1) Inequalities (1.6) and (1.8) indicate in a weak version that order parameters concentrate near the maximum points of curvature within a tangential distance of order $\mathrm{O}\left(\kappa^{-1 / 6}\right)$. We believe that the concentration is within a tangential distance of order $\mathrm{O}\left(\kappa^{-1 / 2}\right)$ to the maximum points of curvature.

(2) We believe that, under the condition $0<H_{C_{3}}(\kappa)-\sigma<L_{1} \kappa^{-1 / 3}$, it holds that

$$
\|\psi\|_{L^{\infty}(\Omega)}=\mathrm{O}\left(\left[H_{C_{3}}(\kappa)-\sigma\right]^{1 / 2} \kappa^{-1 / 2}\right) .
$$

Moreover, if $\mathcal{N}(\partial \Omega)$ consists of isolated points, then

$$
\frac{\|\psi\|_{L^{2}(\Omega)}}{\|\psi\|_{L^{\infty}(\Omega)}}=\mathrm{O}\left(\kappa^{-3 / 4}\right)
$$

For a related discussion see Remark 2.6.

Remark 1.7. - Theorems 1.2 and 1.3 are consequences of Theorem 6.1, and Theorem 1.1 is a consequence of Propositions 2.3 and 5.1. We will see that the proofs involve complicated estimates. We have to mention that one difficulty of the problem comes from the fact that the bottom $\beta_{0}$ of the spectrum of the eigenvalue problem (2.6) on the half plane, which is the limit equation of the linearization of the Ginzburg-Landau system (2.2) at the trivial solution $(0, \mathbf{F})$, is not isolated. In fact, the spectrum of (2.6) fills the interval $\left[\beta_{0}, \infty\right)$. 
Remark 1.8. - Combining the arguments in this paper together with the results in [15] (Section 9), we can deal with non-homogeneous magnetic fields. We can improve the estimates of $\sigma^{*}\left(\kappa, H_{0}\right)$ (see [19]) with better error terms, and prove the localizations of the order parameters. Since the discussions will be parallel, we omit the details.

Remark 1.9. - The problem in 3-dimension is less understood. It was proved in [20] that, for a bounded 3-dimensional sample with smooth surface, under a homogeneous magnetic field, superconductivity nucleates first at a portion of surface which is tangential to the applied field. If the tangential portion is a 1- or 2-dimensional submanifold of the surface, it would be interesting to see what replace the curvature in this case.

The outline of this paper is the following. In Section 2, we improve the lower bound estimate for $H_{C_{3}}$ by getting an error term in $\mathrm{O}\left(\kappa^{-1 / 2}\right)$. In Section 3, we establish a uniform estimate for the lowest eigenvalue of a Schrödinger operator with a magnetic field. Section 4 is devoted to the elliptic estimates on the minimal solutions of the Ginzburg-Landau system. An upper bound estimate for $H_{C_{3}}$ is proved in Section 5, using results from Sections 3 and 4. Combining the estimates in Sections 2 and 5 we prove Theorem 1.1. The location of the order parameters when the applied field is close to $H_{C_{3}}$ is discussed in Section 6 and their exponential decay within boundary is established using Agmon's idea [1] (see also [13] for a presentation of the corresponding HelfferSjöstrand techniques in the semi-classical context).

\section{Lower bound estimate of $\boldsymbol{H}_{C_{3}}$ revisited}

In this section we give a lower bound estimate of $H_{C_{3}}$ with an improved error term.

As mentioned in Section 1, we only consider throughout this paper homogeneous applied fields, that is satisfying $\mathcal{H} \equiv \sigma \mathbf{e}_{3}$, where $\sigma$ is a constant, see (1.2). We shall treat $\sigma$ as a large parameter. In order to make our discussion clear, we set

$$
\mathcal{A}=\sigma \mathbf{A}, \quad \varepsilon=\frac{1}{\sqrt{\kappa \sigma}} .
$$

It is more convenient to consider a rescaled Ginzburg-Landau functional defined on $\mathcal{W}$ as follows:

$$
\mathcal{J}(\psi, \mathbf{A})=\int_{\Omega}\left\{\left|\nabla_{\frac{1}{\varepsilon^{2}} \mathbf{A}} \psi\right|^{2}+\frac{1}{\varepsilon^{4}}|\operatorname{curl} \mathbf{A}-1|^{2}-\kappa^{2}|\psi|^{2}+\frac{\kappa^{2}}{2}|\psi|^{4}\right\} \mathrm{d} x,
$$

where

$$
\mathcal{W}=W^{1,2}(\Omega, \mathbb{C}) \times W^{1,2}\left(\Omega, \mathbb{R}^{2}\right) .
$$

Here $W^{1,2}(\Omega, \mathbb{C})$ is the Sobolev space of all complex-valued functions, and $W^{1,2}\left(\Omega, \mathbb{R}^{2}\right)$ is the Sobolev space of all vector-valued functions. Set

$$
E(\kappa, \varepsilon)=\inf _{(\psi, \mathbf{A}) \in \mathcal{W}} \mathcal{J}(\psi, \mathbf{A}) .
$$


Let $(\psi, \mathbf{A})$ be a minimizer of the functional $\mathcal{J}$. Then, $(\psi, \mathbf{A})$ satisfies the Euler equation (see (1.1))

$$
\begin{cases}-\nabla_{\frac{1}{\varepsilon^{2}} \mathbf{A}}^{2} \psi=\kappa^{2}\left(1-|\psi|^{2}\right) \psi, & \\ \operatorname{curl}^{2}(\mathbf{A}-\mathbf{F})=\varepsilon^{2} \Im\left(\bar{\psi} \nabla_{\frac{1}{\varepsilon^{2}} \mathbf{A}} \psi\right), & \text { in } \Omega, \\ \left(\nabla_{\frac{1}{\varepsilon^{2}}} \mathbf{A}\right) \cdot v=0, & \text { on } \partial \Omega, \\ \operatorname{curl}(\mathbf{A}-\mathbf{F})=0, & \end{cases}
$$

where $\mathbf{F}$ is the unique vector field satisfying (1.3). We recall that the minimizer is called a minimal solution of (2.2). Note that (2.2) has two parameters $\kappa$ and $\varepsilon$. However, for simplicity we will denote a minimal solution by $\left(\psi^{\varepsilon}, \mathbf{A}^{\varepsilon}\right)$. Due to the gauge invariance of the Ginzburg-Landau system (2.2), we may always assume that

$$
\operatorname{div} \mathbf{A}^{\varepsilon}=0 \quad \text { in } \Omega, \quad \mathbf{A}^{\varepsilon} \cdot v=0 \quad \text { on } \partial \Omega .
$$

Denote by $\mu=\mu(b \mathbf{A})$ the first eigenvalue of the following eigenvalue problem

$$
\begin{cases}-\nabla_{b \mathbf{A}}^{2} \phi=\mu \phi & \text { in } \Omega, \\ \left(\nabla_{b \mathbf{A}} \phi\right) \cdot v=0 & \text { on } \partial \Omega,\end{cases}
$$

where $b$ is a real number. The following lemma was given in [19] (Lemma 2.1).

LEMMA 2.1. - Let $\mathcal{J}$ be the functional defined in (2.1).

(1) If $\mu\left(\frac{1}{\varepsilon^{2}} \mathbf{F}\right)<\kappa^{2}$, then $\mathcal{J}$ has a non-trivial minimizer.

(2) If $\mathcal{J}$ has a non-trivial minimizer $\left(\psi^{\varepsilon}, \mathbf{A}^{\varepsilon}\right)$, then $\mu\left(\frac{1}{\varepsilon^{2}} \mathbf{A}^{\varepsilon}\right)<\kappa^{2}$.

The proof of Lemma 2.1 is obvious. Note that $(0, \mathbf{F})$ is a trivial solution of the Ginzburg-Landau system with energy $\mathcal{J}(0, \mathbf{F})=0$. If $E(\kappa, \varepsilon)<0$, then $\mathcal{J}$ has a nontrivial minimizer.

However, Lemma 2.1 is our starting point to estimate $H_{C_{3}}$, see [19]. In this section, as in [19], we shall use Lemma 2.1 to obtain the lower bound estimate of $H_{C_{3}}$ with an improved control of the error term.

Let us first recall some facts about an eigenvalue variation problem. For every fixed $z$, let $\beta(z)$ denote the first eigenvalue of the following self-adjoint eigenvalue problem in $L^{2}\left(\mathbb{R}_{+}\right)$:

$$
\left\{\begin{array}{l}
-u^{\prime \prime}+(z+t)^{2} u=\beta(z) u \text { for } t>0, \\
u^{\prime}(0)=0 .
\end{array}\right.
$$

LEMMA 2.2. - There is a unique $z_{0}, z_{0}<0$, such that

$$
\beta_{0} \equiv \inf _{z \in \mathbb{R}^{1}} \beta(z)=\beta\left(z_{0}\right)=z_{0}^{2} .
$$

Moreover, $0.5<\beta_{0}<0.76$.

Lemma 2.2 was proved by Dauge and Helffer in [7], see also [4]. For a different proof see [17]. For the estimate $0.5<\beta_{0}<0.76$ see [19] (Proposition 2.4). Here we 
use the notations from $[17,19]$. In the following we denote by $u(t)$ a unique positive eigenfunction of (2.5) for $z=z_{0}$ and $\beta=\beta_{0}$ (we may choose $u$ such that $u(0)=1$ ). Recall that $u$ has the following property:

$$
\int_{0}^{+\infty}\left(z_{0}+t\right) u^{2}(t) \mathrm{d} t=0 .
$$

Lemma 2.2 is useful in the classification of bounded solutions associated with the bottom of the spectrum of the Neumann realization of the Schrödinger operator $-\nabla_{\omega}^{2}$ in the half plane $\mathbb{R}_{+}^{2}=\left\{\left(x_{1}, x_{2}\right): x_{2}>0\right\}$

$$
\begin{cases}-\nabla_{\omega}^{2} \phi=\beta_{0} \phi & \text { in } \mathbb{R}_{+}^{2} \\ \nabla_{\omega} \phi \cdot v=0 & \text { on } \partial \mathbb{R}_{+}^{2}\end{cases}
$$

where $\omega(x)=\frac{1}{2}\left(-x_{2}, x_{1}\right)$. It was proved in [17] that, the bottom of the spectrum is the number $\beta_{0}$ given in Lemma 2.2, and the bounded solutions of (2.6) associated with $\beta_{0}$ are given by $\phi=c \exp \left(\frac{i x_{1} x_{2}}{2}+i z_{0} x_{1}\right) u\left(x_{2}\right)$.

In the following, we set

$$
C_{1}=\frac{u^{2}(0)}{3\|u\|_{L^{2}\left(\mathbb{R}_{+}\right)}^{2}} .
$$

It is clear from this formula that $C_{1}>0$.

PROPOSITION 2.3 (Lower bound estimate for $H_{C_{3}}$ ). - Assume that $\Omega$ is a bounded 2-dimensional domain of class $C^{4}$. For $\kappa$ large, we have

$$
H_{C_{3}}(\kappa) \geqslant \frac{\kappa}{\beta_{0}}+\frac{C_{1}}{\beta_{0}^{3 / 2}} \kappa_{\max }+\mathrm{O}\left(\kappa^{-1 / 2}\right),
$$

where $C_{1}$ is the positive constant defined in (2.7).

Proof. - Here we need the upper bound of the lowest eigenvalue $\mu\left(\frac{1}{\varepsilon^{2}} \mathbf{F}\right)$ of the Schrödinger operator with constant magnetic field: $\operatorname{curl} \mathbf{F} \equiv 1$, with an error term in $\mathrm{O}\left(\varepsilon^{-1 / 2}\right)$, which was obtained by Helffer and Morame in [15] (Proposition 10.7):

$$
\mu\left(\frac{1}{\varepsilon^{2}} \mathbf{F}\right) \leqslant \frac{1}{\varepsilon^{2}}\left\{\beta_{0}-C_{1} \kappa_{\max } \varepsilon+M \varepsilon^{3 / 2}\right\} .
$$

Using the argument of the appendix in [19], (2.8) follows from (2.9). For reader's convenience we include a proof below, which also gives an upper bound of the energy.

For $M$ introduced in (2.9), we choose $L$ such that $L \beta_{0}^{5 / 4}>M$. We shall show that, when $\kappa$ is large, (2.2) has a non-trivial minimal solution for any number $\sigma$ satisfying

$$
0<\sigma<\frac{\kappa}{\beta_{0}}+\frac{C_{1}}{\beta_{0}^{3 / 2}} \kappa_{\max }-L \kappa^{-1 / 2}
$$


From the previous lower bound estimate obtained for $H_{C_{3}}(\kappa)$ in [19], we know already that (2.2) has non-trivial minimal solutions for $\sigma \leqslant \kappa / \beta_{0}$ when $\kappa$ is large. Hence we only need to consider $\sigma$ 's greater than $\kappa / \beta_{0}$. In the following we choose $\sigma$ such that

$$
\frac{\kappa}{\beta_{0}}<\sigma<\frac{\kappa}{\beta_{0}}+\frac{C_{1}}{\beta_{0}^{3 / 2}} \kappa_{\max }-L \kappa^{-1 / 2} .
$$

An easy computation gives

$$
\beta_{0}>\frac{\kappa}{\sigma}>\beta_{0}-\frac{C_{1} \beta_{0}^{1 / 2}}{\kappa} \kappa_{\max }+L \beta_{0}^{2} \kappa^{-3 / 2}+\mathrm{O}\left(\kappa^{-2}\right) .
$$

Recall that $\varepsilon=(\sigma \kappa)^{-1 / 2}$. From (2.11) we see that

$$
\frac{\beta_{0}^{1 / 2}}{\kappa}>\varepsilon>\frac{\beta_{0}^{1 / 2}}{\kappa}-\frac{C_{1}}{2} \kappa_{\max } \kappa^{-2}+\frac{L \beta_{0}^{3 / 2}}{2} \kappa^{-5 / 2}+\mathrm{O}\left(\kappa^{-3}\right) .
$$

Hence we have

$$
\begin{aligned}
& \frac{\varepsilon}{\beta_{0}^{1 / 2}}<\frac{1}{\kappa}<\frac{\varepsilon}{\beta_{0}^{1 / 2}}+\frac{C_{1}}{2 \beta_{0}^{3 / 2}} \kappa_{\max } \varepsilon^{2}+\mathrm{O}\left(\varepsilon^{5 / 2}\right), \\
& \beta_{0}>\frac{\kappa}{\sigma}>\beta_{0}-C_{1} \kappa_{\max } \varepsilon+L \beta_{0}^{5 / 4} \varepsilon^{3 / 2}+\mathrm{O}\left(\varepsilon^{2}\right) .
\end{aligned}
$$

Let us write

$$
t(\varepsilon)=\varepsilon^{2}\left(\kappa^{2}-\mu\left(\frac{1}{\varepsilon^{2}} \mathbf{F}\right)\right) .
$$

Using (2.9), (2.11) and (2.12), we have, as $\varepsilon \rightarrow 0$,

$$
t(\varepsilon)=\varepsilon^{2}\left(\kappa^{2}-\mu\left(\frac{1}{\varepsilon^{2}} \mathbf{F}\right)\right) \geqslant\left(L \beta_{0}^{5 / 4}-M\right) \varepsilon^{3 / 2}+\mathrm{O}\left(\varepsilon^{2}\right)>0 .
$$

Let $\phi_{\varepsilon}$ be an eigenfunction of $-\nabla_{\frac{1}{\varepsilon^{2}} \mathbf{F}}^{2}$ in $\Omega$ associated with the first eigenvalue $\mu\left(\frac{1}{\varepsilon^{2}} \mathbf{F}\right)$ of the Neumann realization of $-\nabla_{\frac{1}{\varepsilon^{2}} \mathbf{F}}^{2}$ and choose $\left(\lambda \phi_{\varepsilon}, \mathbf{F}\right)$ as a test function, where $\lambda$ is a free parameter. We have

$$
\begin{aligned}
\mathcal{J}\left(\lambda \phi_{\varepsilon}, \mathbf{F}\right) & =\lambda^{2} \int_{\Omega}\left\{\left|\nabla_{\frac{1}{\varepsilon^{2}} \mathbf{F}} \phi_{\varepsilon}\right|^{2}-\kappa^{2}\left|\phi_{\varepsilon}\right|^{2}+\frac{\lambda^{2} \kappa^{2}}{2}\left|\phi_{\varepsilon}\right|^{4}\right\} \mathrm{d} x \\
& =-\frac{\lambda^{2} t(\varepsilon)}{\varepsilon^{2}}\left\|\phi_{\varepsilon}\right\|_{L^{2}(\Omega)}^{2}+\frac{\lambda^{4} \kappa^{2}}{2}\left\|\phi_{\varepsilon}\right\|_{L^{4}(\Omega)}^{4} .
\end{aligned}
$$

Choose

$$
\lambda^{2}=\frac{t(\varepsilon)\left\|\phi_{\varepsilon}\right\|_{L^{2}(\Omega)}^{2}}{\varepsilon^{2} \kappa^{2}\left\|\phi_{\varepsilon}\right\|_{L^{4}(\Omega)}^{4}}=\frac{t(\varepsilon) \sigma}{\kappa} \frac{\left\|\phi_{\varepsilon}\right\|_{L^{2}(\Omega)}^{2}}{\left\|\phi_{\varepsilon}\right\|_{L^{4}(\Omega)}^{4}} .
$$


Then

$$
\mathcal{J}\left(\lambda \phi_{\varepsilon}, \mathbf{F}\right)=-\frac{t(\varepsilon)^{2}\left\|\phi_{\varepsilon}\right\|_{L^{2}(\Omega)}^{4}}{2 \varepsilon^{4} \kappa^{2}\left\|\phi_{\varepsilon}\right\|_{L^{4}(\Omega)}^{4}}=-\frac{t(\varepsilon)^{2} \sigma^{2}}{2} \frac{\left\|\phi_{\varepsilon}\right\|_{L^{2}(\Omega)}^{4}}{\left\|\phi_{\varepsilon}\right\|_{L^{4}(\Omega)}^{4}}<0 .
$$

This last inequality shows that, for $\sigma$ satisfying (2.10), the Ginzburg-Landau system (2.2) has a non-trivial minimal solution $\left(\psi^{\varepsilon}, \mathbf{A}^{\varepsilon}\right)$. Thus (2.8) holds.

Remark 2.4. - Let $\phi_{\varepsilon}$ and $\lambda$ be the same as in the proof of Proposition 2.3. Note that $\left(\lambda \phi_{\varepsilon}, \mathbf{F}\right)$ is not a solution of (2.2) (a proof is given below for reader's convenience). From the last part of the proof of Proposition 2.3, we have

$$
\mathcal{J}\left(\psi^{\varepsilon}, \mathbf{A}^{\varepsilon}\right)<\mathcal{J}\left(\lambda \phi_{\varepsilon}, \mathbf{F}\right) .
$$

Hence

$$
\mathcal{J}\left(\psi^{\varepsilon}, \mathbf{A}^{\varepsilon}\right)<-\frac{t(\varepsilon)^{2} \sigma^{2}}{2} \frac{\left\|\phi_{\varepsilon}\right\|_{L^{2}(\Omega)}^{4}}{\left\|\phi_{\varepsilon}\right\|_{L^{4}(\Omega)}^{4}} .
$$

Now we show that, for any $\lambda \neq 0,\left(\lambda \phi_{\varepsilon}, \mathbf{F}\right)$ is not a solution of (2.2). Otherwise, suppose that $\left(\lambda \phi_{\varepsilon}, \mathbf{F}\right)$ were a solution of (2.2). We have

$$
\begin{cases}-\nabla_{\frac{1}{\varepsilon^{2}} \mathbf{F}}^{2} \phi_{\varepsilon}=\kappa^{2}\left(1-\lambda^{2}\left|\phi_{\varepsilon}\right|^{2}\right) \phi_{\varepsilon}, & \\ \Im\left(\bar{\phi}_{\varepsilon} \nabla_{\frac{1}{\varepsilon^{2}} \mathbf{F}} \phi_{\varepsilon}\right)=0 & \text { in } \Omega, \\ l\left(\nabla_{\frac{1}{\varepsilon^{2}} \mathbf{F}} \phi_{\varepsilon}\right) \cdot v=0 & \text { on } \partial \Omega .\end{cases}
$$

Recall that $\phi_{\varepsilon}$ satisfies

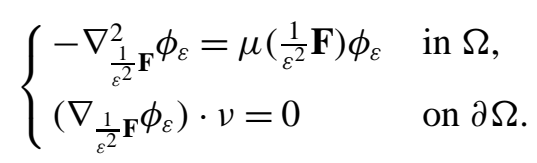

Comparing the first equation of $\left(2.2^{\prime}\right)$ with $(2.15)$ we see that

$$
\mu\left(\frac{1}{\varepsilon^{2}} \mathbf{F}\right) \phi_{\varepsilon}=\kappa^{2}\left(1-\lambda^{2}\left|\phi_{\varepsilon}\right|^{2}\right) \phi_{\varepsilon}
$$

Since $\phi_{\varepsilon} \not \equiv 0$, we have

$$
\left|\phi_{\varepsilon}(x)\right|^{2} \equiv \frac{\kappa^{2}-\mu\left(\frac{1}{\varepsilon^{2}} \mathbf{F}\right)}{\kappa^{2} \lambda^{2}} .
$$

Therefore $\left|\phi_{\varepsilon}(x)\right| \equiv f_{\varepsilon}$, a constant. Let $D$ be a simply-connected subdomain of $\Omega$. In $D$ we can write $\phi_{\varepsilon}(x)=f_{\varepsilon} \mathrm{e}^{i \chi_{\varepsilon}(x)}$, where $\chi_{\varepsilon}(x)$ is a real-valued $C^{2}$ function. Thus

$$
\Im\left(\overline{\phi_{\varepsilon}} \nabla_{\frac{1}{\varepsilon^{2}} \mathbf{F}} \phi_{\varepsilon}\right)=\mathfrak{s}\left[f_{\varepsilon} \mathrm{e}^{-i \chi_{\varepsilon}} \nabla_{\frac{1}{\varepsilon^{2}} \mathbf{F}}\left(f_{\varepsilon} \mathrm{e}^{i \chi_{\varepsilon}}\right)\right]=f_{\varepsilon}^{2}\left(\nabla \chi_{\varepsilon}-\frac{1}{\varepsilon^{2}} \mathbf{F}\right) .
$$


Hence $\mathbf{F}=\varepsilon^{2} \nabla \chi_{\varepsilon}$ and $\operatorname{curl} \mathbf{F} \equiv 0$ in $D$. Repeating this argument on any simplyconnected subdomain of $\Omega$, we find $\operatorname{curl} \mathbf{F} \equiv 0$ in $\Omega$, which contradicts (1.3). This contradiction shows that $\left(\lambda \phi_{\varepsilon}, \mathbf{F}\right)$ is not a solution of (2.2).

Remark 2.5. - Bernoff and Sternberg [3] gave a formal expansion of $H_{C_{3}}(\kappa)$.

It was proved in [19] that, if $\Omega$ is a bounded domain in $\mathbb{R}^{2}$ of class $C^{5}$ and if $\mathbf{F} \in C^{5}(\Omega)$ satisfying $\operatorname{curl} \mathbf{F} \equiv 1$, then, as $\varepsilon \rightarrow 0$, we have

$$
\mu\left(\frac{1}{\varepsilon^{2}} \mathbf{F}\right) \leqslant \frac{1}{\varepsilon^{2}}\left\{\beta_{0}-C_{1} \kappa_{\max } \varepsilon+\mathrm{O}\left(\varepsilon^{8 / 7}\right)\right\} .
$$

From this estimate Lu-Pan derived a lower bound estimate of $H_{C_{3}}$ with error term in $\mathrm{O}\left(\kappa^{-1 / 7}\right)$.

In [9] del Pino, Felmer and Sternberg proved, for a $C^{3, \alpha}$ domain, an upper bound of the lowest eigenvalue with a remainder in $o(1 / \varepsilon)$, namely

$$
\mu\left(\frac{1}{\varepsilon^{2}} \mathbf{F}\right) \leqslant \frac{1}{\varepsilon^{2}}\left\{\beta_{0}-C_{1} \kappa_{\max } \varepsilon+\mathrm{o}(\varepsilon)\right\},
$$

which yields a lower bound estimate for $H_{C_{3}}(\kappa)$ with error term o(1). They also pointed out that, under the stronger assumption that there is a non-degenerate maximum point $z\left(s^{*}\right)$ of the curvature, they could prove an upper bound of the lowest eigenvalue in the form

$$
\mu\left(\frac{1}{\varepsilon^{2}} \mathbf{F}\right) \leqslant \frac{1}{\varepsilon^{2}}\left\{\beta_{0}-C_{1} \kappa_{\max } \varepsilon+\frac{\beta_{0}^{1 / 4}}{\sqrt{6}} \sqrt{-\kappa_{r}^{\prime \prime}\left(s^{*}\right)} \varepsilon^{3 / 2}+\mathrm{o}\left(\varepsilon^{3 / 2}\right)\right\} .
$$

We should also mention that the expression of $C_{1}$ in [19] is more complicated (and equivalent). Helffer and Morame in [15] used $2 M_{3}$ instead of $C_{1}$. In this paper we adopt the definition (2.7) for $C_{1}$, as in [3], which shows more explicitly the positivity of $C_{1}$.

Remark 2.6. - The energy estimate given in (2.14) involves the term $\left\|\phi_{\varepsilon}\right\|_{L^{2}(\Omega)}^{4} /$ $\left\|\phi_{\varepsilon}\right\|_{L^{4}(\Omega)}^{4}$ which, we believe, is of order $\mathrm{O}\left(\varepsilon^{3 / 2}\right)$ if $\mathcal{N}(\partial \Omega)$ consists of a finite number of points.

We explain the guess heuristically. For simplicity, let us consider the normalized eigenfunction $\phi_{\varepsilon}$, namely, $\left\|\phi_{\varepsilon}\right\|_{L^{\infty}(\Omega)}=1$. From Propositions 6.6, 10.6 and 10.7 in [15] we believe (and will show later) that the eigenfunction $\phi_{\varepsilon}$ concentrates on the set $\mathcal{N}(\partial \Omega)$ of the maximum points of the curvature, and decays exponentially away from $\mathcal{N}(\partial \Omega)$, with order $\exp \left(-\frac{\alpha_{1}}{\varepsilon} \operatorname{dist}(x, \partial \Omega)\right)$ in the normal direction, and with order $\exp \left(-\frac{\alpha_{2}}{\sqrt{\varepsilon}} d_{t}(x, \mathcal{N}(\partial \Omega))\right)$ in the tangential direction.

Now suppose that $\mathcal{N}(\partial \Omega)$ consists of a finite number of points. Near each point $x_{j} \in \mathcal{N}(\partial \Omega)$, we can introduce new variables $y=\left(y_{1}, y_{2}\right)$, where $y_{1}$ is the tangential variable and $y_{2}$ is the normal variable, to straighten a portion of the boundary around $x_{j}$ (in fact, $y_{1}$ is a translation of $s(x)$, and $y_{2}=t(x)$ ). In the new variables $y$, after making gauge transformations, the rescaled function of $\phi_{\varepsilon}$ converges to a bounded solution $c_{j} \phi_{0}(y)$ of (2.6) as $\varepsilon \rightarrow 0$, where $\left|c_{j}\right| \leqslant 1, \phi_{0}(y)=\exp \left(i z_{0} y_{1}\right) u\left(y_{2}\right)$ and $u$ is chosen such that $u(0)=1$ (recall that $\left\|\phi_{\varepsilon}\right\|_{L^{\infty}(\Omega)}=1$ ). So, near $x_{j}, \phi_{\varepsilon}$ can be approximated 
locally by a function in the form $\mathrm{e}^{i \chi_{j}} \phi_{0}(y / \varepsilon) w_{j}\left(y_{1} / \sqrt{\varepsilon}\right)$, here $\chi_{j}$ and $w_{j}$ are smooth real functions, and $\left|w_{j}(y)\right| \leqslant 1$. Hence

$$
\int_{\Omega \cap B_{\delta}\left(x_{j}\right)}\left|\phi_{\varepsilon}\right|^{2} \mathrm{~d} x \simeq \hat{c}_{j} \varepsilon^{3 / 2} .
$$

As mentioned above that $\phi_{\varepsilon}$ concentrates on $\mathcal{N}(\partial \Omega)$. The above computation can be carried out at each of the points $x_{1}, \ldots, x_{n}$ in $\mathcal{N}(\partial \Omega)$. Hence

$$
\int_{\Omega}\left|\phi_{\varepsilon}\right|^{2} \mathrm{~d} x \simeq \varepsilon^{3 / 2} \sum_{j=1}^{n} \hat{c}_{j}, \quad \sum_{j=1}^{n} \hat{c}_{j}^{2}>0 .
$$

Similarly we have

$$
\int_{\Omega}\left|\phi_{\varepsilon}\right|^{4} \mathrm{~d} x \simeq \varepsilon^{3 / 2} \sum_{j=1}^{n} \tilde{c}_{j}, \quad \sum_{j=1}^{n} \tilde{c}_{j}^{2}>0 .
$$

Therefore

$$
\frac{\left\|\phi_{\varepsilon}\right\|_{L^{2}(\Omega)}^{4}}{\left\|\phi_{\varepsilon}\right\|_{L^{4}(\Omega)}^{4}} \simeq c \varepsilon^{3 / 2}, \quad \text { where } c=\frac{\left[\sum_{j=1}^{n} \hat{c}_{j}\right]^{2}}{\sum_{j=1}^{n} \tilde{c}_{j}} .
$$

Remark 2.7. - Instead of using the eigenfunction $\phi_{\varepsilon}$, we can also choose the test function used in [15] (proof of Proposition 10.7) to get a more explicit energy estimate.

\section{Uniform estimates for the lowest eigenvalue}

In this section we assume that $\Omega$ is a bounded simply-connected domain in $\mathbb{R}^{2}$ of class $C^{4}$. We shall establish a uniform estimate for the lowest eigenvalue $\mu_{\varepsilon} \equiv \mu\left(\frac{1}{\varepsilon^{2}} \mathbf{A}^{\varepsilon}\right)$ of the Neumann realization of the Schrödinger operator $-\nabla_{\frac{1}{\varepsilon^{2}}}^{2} \mathbf{A}^{\varepsilon}$ with magnetic potential $\frac{1}{\varepsilon^{2}} \mathbf{A}^{\varepsilon}$, where $\mathbf{A}^{\varepsilon}$ satisfies the following conditions

$$
\begin{aligned}
& \left\|\mathbf{A}^{\varepsilon}\right\|_{C^{2}(\bar{\Omega})} \leqslant C, \\
& \left\|\operatorname{curl} \mathbf{A}^{\varepsilon}-1\right\|_{C^{1}(\bar{\Omega})}=\mathrm{O}\left(\varepsilon^{1 / 3}\right), \quad \text { as } \varepsilon \rightarrow 0, \\
& \operatorname{curl} \mathbf{A}^{\varepsilon}=1, \quad \text { on } \partial \Omega .
\end{aligned}
$$

We intend to apply the results of this section to the solution $\mathbf{A}^{\varepsilon}$ of (2.2) in order to prove Theorem 1 . However, the results in this section are valid for any family of vector fields satisfying (3.1).

THEOREM 3.1. - Under assumption (3.1), there exist $\varepsilon_{0}>0$ and $C>0$ such that, for all $0<\varepsilon<\varepsilon_{0}$,

$$
\mu\left(\frac{1}{\varepsilon^{2}} \mathbf{A}^{\varepsilon}\right) \geqslant \frac{1}{\varepsilon^{2}}\left(\beta_{0}-C_{1} \kappa_{\max } \varepsilon-C \varepsilon^{4 / 3}\right),
$$

where $C_{1}$ is given in (2.7). 
The proof of Theorem 3.1 will be carried out following the lines of [15], with a careful control on the error terms. In this section we always fix a positive number $\delta_{0}<\varepsilon(\Omega)$, and let $t(x)=\operatorname{dist}(x, \partial \Omega)$. By the gauge-invariance of the eigenvalue problem and condition (3.1), we can choose $\mathbf{A}^{\varepsilon}$ in such a way that, in the neighborhood $\Omega_{\delta_{0}}$, $\mathbf{A}^{\varepsilon}=\left(A_{1}^{\varepsilon}, A_{2}^{\varepsilon}\right)$ has the following form:

$$
\begin{aligned}
& A_{1}^{\varepsilon}=-t+\frac{t^{2}}{2} \kappa_{r}(s)+t^{2} b_{\varepsilon}(s, t), \\
& A_{2}^{\varepsilon}=0, \\
& b_{\varepsilon}(s, t)=\mathrm{O}\left(\varepsilon^{1 / 3}\right) .
\end{aligned}
$$

Let $\left\{\chi_{\gamma}(x)\right\}_{\Gamma}$ be a partition of unity of $\mathbb{R}^{2}$ introduced in [15] ((9.10)-(9.14)) such that $\Gamma=\mathbb{Z}^{2}$,

$$
\begin{aligned}
& \chi_{\gamma} \in C^{\infty}\left(\mathbb{R}^{2}, \mathbb{R}\right), \\
& \operatorname{spt}\left(\chi_{\gamma}\right) \subset \gamma+[-1,1]^{2}, \quad \text { for any } \gamma \in \Gamma, \\
& \sum_{\gamma \in \Gamma} \chi_{\gamma}(x)^{2}=1, \quad \sum_{\gamma \in \Gamma}\left|\nabla \chi_{\gamma}(x)\right|^{2}<C .
\end{aligned}
$$

If $\tau(\varepsilon)$ is a function of $\varepsilon$ such that $0<\tau(\varepsilon)<\delta_{0}$ for all $\varepsilon$ small, then we set

$$
\chi_{\gamma, \tau(\varepsilon)}(x)=\chi_{\gamma}\left(\frac{x}{\tau(\varepsilon)}\right), \quad \text { for all } \gamma \in \Gamma .
$$

Thus we have a new partition of unity such that

$$
\begin{aligned}
& \operatorname{spt}\left(\chi_{\gamma, \tau(\varepsilon)}\right) \subset \tau(\varepsilon) \gamma+[-\tau(\varepsilon), \tau(\varepsilon)]^{2}, \\
& \sum_{\gamma \in \Gamma} \chi_{\gamma, \tau(\varepsilon)}(x)^{2}=1, \quad \sum_{\gamma \in \Gamma}\left|\nabla \chi_{\gamma, \tau(\varepsilon)}(x)\right|^{2}<\frac{C}{\tau(\varepsilon)^{2}} .
\end{aligned}
$$

Let us introduce:

$$
\begin{aligned}
& \Gamma_{\tau(\varepsilon)}(\Omega)=\left\{\gamma \in \Gamma: \operatorname{spt}\left(\chi_{\gamma, \tau(\varepsilon)}\right) \cap \Omega \neq \emptyset\right\}, \\
& \Gamma_{\tau(\varepsilon)}^{0}(\Omega)=\left\{\gamma \in \Gamma_{\tau(\varepsilon)}(\Omega): \operatorname{dist}\left(\operatorname{spt}\left(\chi_{\gamma, \tau(\varepsilon)}\right), \partial \Omega\right)>\tau(\varepsilon)\right\}, \\
& \Gamma_{\tau(\varepsilon)}^{1}(\Omega)=\left\{\gamma \in \Gamma_{\tau(\varepsilon)}(\Omega): \operatorname{dist}\left(\operatorname{spt}\left(\chi_{\gamma, \tau(\varepsilon)}\right), \partial \Omega\right) \leqslant \tau(\varepsilon)\right\}, \\
& \Gamma_{\tau(\varepsilon)}^{\prime}(\Omega)=\left\{\gamma \in \Gamma_{\tau(\varepsilon)}^{1}(\Omega): \operatorname{spt}\left(\chi_{\gamma, \tau(\varepsilon)}\right) \cap \bar{\Omega} \subset \Omega_{\delta_{0}}\right\} .
\end{aligned}
$$

In the following we denote by $\varphi_{\varepsilon}$ an eigenfunction of the Neumann-Schrödinger operator $-\nabla_{\frac{1}{\varepsilon^{2}} \mathbf{A}^{\varepsilon}}^{2}$ associated with the lowest eigenvalue $\mu_{\varepsilon}$. We shall recall the estimates for eigenfunctions. Let us begin with the weighted $L^{2}$ estimates obtained in [15] ((6.25) and (6.26)):

LEMMA 3.2 (Weighted $L^{2}$ estimates). - Under assumption (3.1), there exists positive constants $\alpha, \varepsilon_{1}$ and $C$ such that, for all $0<\varepsilon \leqslant \varepsilon_{1}$, we have

$$
\int_{\Omega} \exp \left(\frac{\alpha t(x)}{\varepsilon}\right)\left\{\left|\varphi_{\varepsilon}\right|^{2}+\varepsilon^{2}\left|\nabla_{\frac{1}{\varepsilon^{2}} \mathbf{A}^{\varepsilon}} \varphi_{\varepsilon}\right|^{2}\right\} \mathrm{d} x \leqslant C \int_{\Omega}\left|\varphi_{\varepsilon}\right|^{2} \mathrm{~d} x .
$$


Propositions 9.6 and 10.2 in [15] remain true for a family $\left\{\mathbf{A}^{\varepsilon}\right\}$ satisfying assumption (3.1).

LEMMA 3.3. - Under assumption (3.1), for any non-negative integer $k$, there exist positive numbers $\varepsilon_{2}(k)$ and $C_{k}$ such that, for any $0<\varepsilon<\varepsilon_{2}(k)$, we have

$$
\begin{aligned}
& \int_{\Omega} t(x)^{k}\left|\varphi_{\varepsilon}(x)\right|^{2} \mathrm{~d} x \leqslant C_{k} \varepsilon^{k}\left\|\varphi_{\varepsilon}\right\|_{L^{2}(\Omega)}^{2}, \\
& \int_{\Omega} t(x)^{k}\left|\nabla_{\frac{1}{\varepsilon^{2}}{ }^{\varepsilon}} \varphi_{\varepsilon}(x)\right|^{2} \mathrm{~d} x \leqslant C_{k} \varepsilon^{k-2}\left\|\varphi_{\varepsilon}\right\|_{L^{2}(\Omega)}^{2} .
\end{aligned}
$$

LEMMA 3.4. - Under assumption (3.1), we choose $\tau(\varepsilon)=\varepsilon^{\rho}$ with $0<\rho \leqslant 1 / 2$. Then, for any non-negative integer $k$, there exist positive numbers $\varepsilon_{3}(k)$ and $C(k)$, and sequences $\left\{a_{k}(\gamma, \varepsilon)\right\}_{\gamma}$ and $\left\{b_{k}(\gamma, \varepsilon)\right\}_{\gamma}$ satisfying

$$
\sum_{\gamma \in \Gamma_{\tau(\varepsilon)}^{\prime}} a_{k}(\gamma, \varepsilon)^{2} \leqslant C(k), \quad \sum_{\gamma \in \Gamma_{\tau(\varepsilon)}^{\prime}} b_{k}(\gamma, \varepsilon)^{2} \leqslant C(k),
$$

such that, for any $0<\varepsilon<\varepsilon_{3}(k)$ and $\gamma \in \Gamma_{\tau(\varepsilon)}^{\prime}(\Omega)$, we have

$$
\begin{aligned}
& \int_{\Omega} t(x)^{k}\left|\chi_{\gamma, \tau(\varepsilon)} \varphi_{\varepsilon}(x)\right|^{2} \mathrm{~d} x \leqslant a_{k}(\gamma, \varepsilon)^{2} \varepsilon^{k}\left\|\varphi_{\varepsilon}\right\|_{L^{2}(\Omega)}^{2}, \\
& \int_{\Omega} t(x)^{k}\left|\nabla_{\frac{1}{\varepsilon^{2}} A^{\varepsilon}}\left(\chi_{\gamma, \tau(\varepsilon)} \varphi_{\varepsilon}(x)\right)\right|^{2} \mathrm{~d} x \leqslant b_{k}(\gamma, \varepsilon)^{2} \varepsilon^{k-2}\left\|\varphi_{\varepsilon}\right\|_{L^{2}(\Omega)}^{2} .
\end{aligned}
$$

Remark 3.5. - The exponential decay in the normal direction is well known in the case where the magnetic field is independent of the parameter $\varepsilon$. Under assumption (3.1), the uniformly exponential decay can also be established, as in [9] (Theorem 4.3) and [15] (Theorem 6.3):

Under assumption (3.1), there exist $\varepsilon_{4}>0$ and, for any multi-index $\alpha$ with $|\alpha| \leqslant 2$, positive constants $c_{1}(\alpha)$ and $c_{2}(\alpha)$ such that, for any $0<\varepsilon<\varepsilon_{4}$, we have

$$
\left|D^{\alpha} \varphi_{\varepsilon}(x)\right| \leqslant \frac{c_{1}(\alpha)}{\varepsilon^{|\alpha|}} \exp \left(-\frac{c_{2}(\alpha)}{\varepsilon} \operatorname{dist}(x, \partial \Omega)\right)\left\|\varphi_{\varepsilon}\right\|_{L^{\infty}(\Omega)}, \quad \text { for all } x \in \Omega .
$$

Proof of Theorem 3.1. - In the proof $C$ denotes a generic constant which varies from line to line, but is independent of $\varepsilon$ and $\gamma$. As above, we denote by $\varphi_{\varepsilon}$ an eigenfunction associated with the lowest eigenvalue $\mu_{\varepsilon}$. Write

$$
Q_{\varepsilon^{2}, \mathbf{A}}[\phi]=\int_{\Omega}\left|\nabla_{\frac{1}{\varepsilon^{2}} \mathbf{A}} \phi\right|^{2} \mathrm{~d} x .
$$

Then

$$
\mu_{\varepsilon}=\frac{Q_{\varepsilon^{2}, \mathbf{A}^{\varepsilon}}\left[\varphi_{\varepsilon}\right]}{\left\|\varphi_{\varepsilon}\right\|_{L^{2}(\Omega)}^{2}}
$$


So, a lower bound of $Q_{\varepsilon^{2}, \mathbf{A}^{\varepsilon}}\left[\varphi_{\varepsilon}\right]$ yields a lower bound for $\mu_{\varepsilon}$. However, we will not compute directly the value of $Q_{\varepsilon^{2}, \mathbf{A}^{\varepsilon}}\left[\varphi_{\varepsilon}\right]$. Recall that the eigenfunctions concentrate in a small neighborhood of the boundary. Also note that, for any smooth function $\eta$, we have

$$
\int_{\Omega}\left|\nabla_{\frac{1}{\varepsilon^{2}} \mathbf{A}^{\varepsilon}}\left(\eta \varphi_{\varepsilon}\right)\right|^{2} \mathrm{~d} x=\Re \int_{\Omega}\left(-\nabla_{\frac{1}{\varepsilon^{2}} \mathbf{A}^{\varepsilon}}^{2} \varphi_{\varepsilon}\right) \overline{\left(\eta^{2} \varphi_{\varepsilon}\right)} \mathrm{d} x+\int_{\Omega}\left|\varphi_{\varepsilon} \nabla \eta\right|^{2} \mathrm{~d} x,
$$

hence

$$
\mu_{\varepsilon}\left\|\varphi_{\varepsilon} \eta\right\|_{L^{2}(\Omega)}^{2}=Q_{\varepsilon^{2}, \mathbf{A}^{\varepsilon}}\left[\varphi_{\varepsilon} \eta\right]-\int_{\Omega}\left|\varphi_{\varepsilon} \nabla \eta\right|^{2} \mathrm{~d} x .
$$

Therefore, we shall choose a suitable cut-off function $\eta$ carefully, and estimate each term in the right hand of (3.9). We shall carry out a careful analysis on the behavior of $\varphi_{\varepsilon}$ near the boundary, and the following notations are needed.

Let $\left\{\eta_{0, \tau(\varepsilon)}, \eta_{1, \tau(\varepsilon)}\right\}$ be the partition of unity on $\mathbb{R}$ introduced in [15] ((9.22) and (9.23)) such that

$$
\begin{aligned}
& \eta_{0, \tau(\varepsilon)}^{2}(t)+\eta_{1, \tau(\varepsilon)}^{2}(t)=1, \\
& \left|\eta_{j, \tau(\varepsilon)}^{\prime}(t)\right| \leqslant \frac{C}{\tau(\varepsilon)}, \quad \text { for } j=0,1, \\
& \operatorname{spt}\left(\eta_{0, \tau(\varepsilon)}\right) \subset\left[\frac{\tau(\varepsilon)}{20},+\infty\right), \\
& \operatorname{spt}\left(\eta_{1, \tau(\varepsilon)}\right) \subset\left(-\infty, \frac{\tau(\varepsilon)}{10}\right] .
\end{aligned}
$$

Let us choose $\tau(\varepsilon)=\varepsilon^{\rho}$, where $1 / 3 \leqslant \rho \leqslant 1 / 2$. We will fix $\rho$ later. For each $\gamma \in$ $\Gamma_{\tau(\varepsilon)}^{1}(\Omega)$, we can choose $x_{\gamma} \in \partial \Omega$, such that, for any $x \in \operatorname{spt}\left(\chi_{\gamma, \varepsilon^{1 / 3}}\right)$

$$
\left|s-s_{\gamma}\right| \leqslant \frac{3}{2} \varepsilon^{1 / 3}
$$

Set

$$
\begin{aligned}
& K(\gamma, \varepsilon)=\left(s_{\gamma}-2 \varepsilon^{1 / 3}, s_{\gamma}+2 \varepsilon^{1 / 3}\right) \times(0, \tau(\varepsilon)), \\
& K_{\gamma}=\kappa_{r}\left(s_{\gamma}\right), \\
& a_{\gamma}(t)=1-K_{\gamma} t .
\end{aligned}
$$

Let us define

$$
\begin{aligned}
& w_{\varepsilon}(x)=\eta_{1, \tau(\varepsilon)}(t(x)) \varphi_{\varepsilon}(x), \\
& w_{\gamma, \varepsilon}(x)=\chi_{\gamma, \varepsilon^{1 / 3}} \eta_{1, \tau(\varepsilon)}(t(x)) \varphi_{\varepsilon}(x) .
\end{aligned}
$$

Using (3.9) with $\eta=\eta_{1, \tau(\varepsilon)}$, we have

$$
\mu_{\varepsilon}\left\|w_{\varepsilon}\right\|_{L^{2}(\Omega)}^{2}=Q_{\varepsilon^{2}, \mathbf{A}^{\varepsilon}}\left[w_{\varepsilon}\right]-\int_{\Omega}\left|\varphi_{\varepsilon} \nabla \eta_{1, \tau(\varepsilon)}\right|^{2} \mathrm{~d} x .
$$


We shall estimate each term in the right hand of (3.12).

Step 1. We look for a lower bound of $Q_{\varepsilon^{2}, \mathbf{A}^{\varepsilon}}\left[w_{\varepsilon}\right]$. As in [15] ((11.3) and (11.4)), we get

$$
Q_{\varepsilon^{2}, \mathbf{A}^{\varepsilon}}\left[w_{\varepsilon}\right] \geqslant \sum_{\gamma \in \Gamma_{\tau(\varepsilon)}^{1}(\Omega)} Q_{\varepsilon^{2}, \mathbf{A}^{\varepsilon}}\left[w_{\gamma, \varepsilon}\right]-C \varepsilon^{-2 / 3} \int_{\Omega}\left|w_{\varepsilon}\right|^{2} \mathrm{~d} x
$$

and

$$
\begin{aligned}
Q_{\varepsilon^{2}, \mathbf{A}^{\varepsilon}}\left[w_{\gamma, \varepsilon}\right] \geqslant & \int_{K(\gamma, \varepsilon)} a_{\gamma}(t)\left\{\left(1+2 K_{\gamma} t\right)\left|\left(\partial_{s}-\frac{i}{\varepsilon^{2}} A_{1}^{\varepsilon}\right) w_{\gamma, \varepsilon}\right|^{2}+\left|\partial_{t} w_{\gamma, \varepsilon}\right|^{2}\right\} \mathrm{d} s \mathrm{~d} t \\
& -C \int_{\Omega}\left\{\varepsilon^{1 / 3} t\left|\nabla_{\frac{1}{\varepsilon^{2}} \mathbf{A}^{\varepsilon}} w_{\gamma, \varepsilon}\right|^{2}+\varepsilon^{-11 / 3}\left(t^{3}+t^{4}\right)\left|w_{\gamma, \varepsilon}\right|^{2}\right\} \mathrm{d} x .
\end{aligned}
$$

From these inequalities, Lemmas 3.4 and 3.5, we get

$$
\begin{aligned}
Q_{\varepsilon^{2}, \mathbf{A}^{\varepsilon}}\left[w_{\varepsilon}\right] \geqslant & \sum_{\gamma \in \Gamma_{\tau(\varepsilon)}^{1}(\Omega)} \int_{K(\gamma, \varepsilon)} a_{\gamma}(t)\left\{\left(1+2 K_{\gamma} t\right)\left|\left(\partial_{s}-\frac{i}{\varepsilon^{2}} A_{1}^{\varepsilon}\right) w_{\gamma, \varepsilon}\right|^{2}\right. \\
& \left.+\left|\partial_{t} w_{\gamma, \varepsilon}\right|^{2}\right\} \mathrm{d} s \mathrm{~d} t-C \varepsilon^{-2 / 3}\left\|w_{\varepsilon}\right\|_{L^{2}(\Omega)}^{2} .
\end{aligned}
$$

In order to estimate the sum in the right hand of (3.13), we write

$$
\begin{aligned}
& A_{1}^{\varepsilon}=A_{\gamma}(t)+A_{\gamma, \varepsilon}, \\
& A_{\gamma}(t)=-t+\frac{t^{2}}{2} K_{\gamma}, \\
& A_{\gamma, \varepsilon}=A_{1}^{\varepsilon}-A_{\gamma}=\frac{t^{2}}{2}\left(\kappa_{r}(s)-K_{\gamma}\right)+t^{2} b_{\varepsilon}(s, t) .
\end{aligned}
$$

We have

$$
\begin{aligned}
& \int_{K(\gamma, \varepsilon)} a_{\gamma}(t)\left(1+2 K_{\gamma} t\right)\left|\left(\partial_{s}-\frac{i}{\varepsilon^{2}} A_{1}^{\varepsilon}\right) w_{\gamma, \varepsilon}\right|^{2} \mathrm{~d} s \mathrm{~d} t \\
& =\int_{K(\gamma, \varepsilon)} a_{\gamma}(t)\left(1+2 K_{\gamma} t\right)\left|\left(\partial_{s}-\frac{i}{\varepsilon^{2}} A_{\gamma}\right) w_{\gamma, \varepsilon}-\frac{i}{\varepsilon^{2}} A_{\gamma, \varepsilon} w_{\gamma, \varepsilon}\right|^{2} \mathrm{~d} s \mathrm{~d} t \\
& =\int_{K(\gamma, \varepsilon)} a_{\gamma}(t)\left(1+2 K_{\gamma} t\right)\left|\left(\partial_{s}-\frac{i}{\varepsilon^{2}} A_{\gamma}\right) w_{\gamma, \varepsilon}\right|^{2} \mathrm{~d} s \mathrm{~d} t \\
& \quad+\frac{2}{\varepsilon^{2}} \Im \int_{K(\gamma, \varepsilon)} a_{\gamma}(t)\left(1+2 K_{\gamma} t\right) A_{\gamma, \varepsilon} \overline{w_{\gamma, \varepsilon}}\left(\partial_{s}-\frac{i}{\varepsilon^{2}} A_{\gamma}\right) w_{\gamma, \varepsilon} \mathrm{d} s \mathrm{~d} t \\
& \quad+\frac{1}{\varepsilon^{4}} \int_{K(\gamma, \varepsilon)} a_{\gamma}(t)\left(1+2 K_{\gamma} t\right)\left|A_{\gamma, \varepsilon} w_{\gamma, \varepsilon}\right|^{2} \mathrm{~d} s \mathrm{~d} t .
\end{aligned}
$$


Hence

$$
Q_{\varepsilon^{2}, \mathbf{A}^{\varepsilon}}\left[w_{\varepsilon}\right] \geqslant P(\varepsilon)+\frac{2 q(\varepsilon)}{\varepsilon^{2}}-C \varepsilon^{-2 / 3}\left\|w_{\varepsilon}\right\|_{L^{2}(\Omega)}^{2},
$$

where

$$
\begin{aligned}
& P(\varepsilon)=\sum_{\gamma \in \Gamma_{\tau(\varepsilon)}^{1}(\Omega)} \int_{K(\gamma, \varepsilon)} a_{\gamma}(t)\left\{\left(1+2 K_{\gamma} t\right)\left|\left(\partial_{s}-\frac{i}{\varepsilon^{2}} A_{\gamma}\right) w_{\gamma, \varepsilon}\right|^{2}+\left|\partial_{t} w_{\gamma, \varepsilon}\right|^{2}\right\} \mathrm{d} s \mathrm{~d} t \\
& q(\varepsilon)=\sum_{\gamma \in \Gamma_{\tau(\varepsilon)}^{1}(\Omega)} q(\gamma, \varepsilon), \\
& q(\gamma, \varepsilon)=\Im \int_{K(\gamma, \varepsilon)} a_{\gamma}(t)\left(1+2 K_{\gamma} t\right) A_{\gamma, \varepsilon} \overline{w_{\gamma, \varepsilon}}\left(\partial_{s}-\frac{i}{\varepsilon^{2}} A_{\gamma}\right) w_{\gamma, \varepsilon} \mathrm{d} s \mathrm{~d} t .
\end{aligned}
$$

We need a lower bound of each term in the right hand of (3.15). A lower bound of $P(\varepsilon)$ can be obtained by using the argument in [15], proof of Theorem 11.1, with the choice $\tau(\varepsilon)=\varepsilon^{5 / 12}$ :

$$
P(\varepsilon) \geqslant \frac{1}{\varepsilon^{2}}\left(\beta_{0}-C_{1} \kappa_{\max } \varepsilon-C \varepsilon^{4 / 3}\right)\left\|w_{\varepsilon}\right\|_{L^{2}(\Omega)}^{2} .
$$

In the following, we choose $\tau(\varepsilon)=\varepsilon^{5 / 12}$ and derive an estimate for $2 q(\varepsilon) / \varepsilon^{2}$. Since $A_{\gamma}=A_{1}^{\varepsilon}-A_{\gamma, \varepsilon}$, we can write

$$
\partial_{s}-\frac{i}{\varepsilon^{2}} A_{\gamma}=\partial_{s}-\frac{i}{\varepsilon^{2}} A_{1}^{\varepsilon}+\frac{i}{\varepsilon^{2}} A_{\gamma, \varepsilon} .
$$

Thus we write

$$
\begin{aligned}
& q(\gamma, \varepsilon)=q_{1}(\gamma, \varepsilon)+q_{2}(\gamma, \varepsilon), \\
& q_{1}(\gamma, \varepsilon)=\Im \int_{K(\gamma, \varepsilon)} a_{\gamma}(t)\left(1+2 K_{\gamma} t\right) A_{\gamma, \varepsilon} \overline{w_{\gamma, \varepsilon}}\left(\partial_{s}-\frac{i}{\varepsilon^{2}} A_{1}^{\varepsilon}\right) w_{\gamma, \varepsilon} \mathrm{d} s \mathrm{~d} t, \\
& q_{2}(\gamma, \varepsilon)=\frac{1}{\varepsilon^{2}} \int_{K(\gamma, \varepsilon)} a_{\gamma}(t)\left(1+2 K_{\gamma} t\right)\left|A_{\gamma, \varepsilon} w_{\gamma, \varepsilon}\right|^{2} \mathrm{~d} s \mathrm{~d} t .
\end{aligned}
$$

We first estimate $q_{1}(\gamma, \varepsilon)$. Noting that $\eta_{1, \tau(\varepsilon)}$ depends only on $t$, using (3.11) we have

$$
\begin{aligned}
q_{1}(\gamma, \varepsilon)= & \Im \int_{K(\gamma, \varepsilon)} a_{\gamma}(t)\left(1+2 K_{\gamma} t\right) \eta_{1, \tau(\varepsilon)}^{2}(t) \\
& \times A_{\gamma, \varepsilon} \overline{\chi_{\gamma, \varepsilon^{1 / 3}} \varphi_{\varepsilon}}\left(\partial_{s}-\frac{i}{\varepsilon^{2}} A_{1}^{\varepsilon}\right)\left(\chi_{\gamma, \varepsilon^{1 / 3}} \varphi_{\varepsilon}\right) \mathrm{d} s \mathrm{~d} t,
\end{aligned}
$$

so

$$
\left|q_{1}(\gamma, \varepsilon)\right| \leqslant C \int_{K(\gamma, \varepsilon)} \eta_{1, \tau(\varepsilon)}^{2}(t)\left|A_{\gamma, \varepsilon} \chi_{\gamma, \varepsilon^{1 / 3}} \varphi_{\varepsilon}\right|\left|\left(\partial_{s}-\frac{i}{\varepsilon^{2}} A_{1}^{\varepsilon}\right)\left(\chi_{\gamma, \varepsilon}^{1 / 3} \varphi_{\varepsilon}\right)\right| \mathrm{d} s \mathrm{~d} t
$$




$$
\begin{aligned}
\leqslant & C\left(\int_{K(\gamma, \varepsilon)} \eta_{1, \tau(\varepsilon)}^{2}(t)\left|A_{\gamma, \varepsilon}\right|^{2}\left|\chi_{\gamma, \varepsilon} 1 / 3 \varphi_{\varepsilon}\right|^{2} \mathrm{~d} s \mathrm{~d} t\right)^{1 / 2} \\
& \times\left(\int_{K(\gamma, \varepsilon)} \eta_{1, \tau(\varepsilon)}^{2}(t)\left|\left(\partial_{s}-\frac{i}{\varepsilon^{2}} A_{1}^{\varepsilon}\right)\left(\chi_{\gamma, \varepsilon^{1 / 3}} \varphi_{\varepsilon}\right)\right|^{2} \mathrm{~d} s \mathrm{~d} t\right)^{1 / 2} .
\end{aligned}
$$

From (3.3), (3.10) and (3.14) we have

$$
\left|A_{\gamma, \varepsilon}\right| \leqslant t^{2}\left(\frac{1}{2}\left|\kappa_{r}(s)-\kappa_{r}\left(s_{\gamma}\right)\right|+\left|b_{\varepsilon}(s, t)\right|\right)=\mathrm{O}\left(\varepsilon^{1 / 3}\right) t^{2}, \quad \text { on } K(\gamma, \varepsilon) .
$$

So we use (3.7) with $\alpha=0,(3.5)$ and (3.6), in order to obtain

$$
\begin{aligned}
& \int_{K(\gamma, \varepsilon)} \eta_{1, \tau(\varepsilon)}^{2}(t)\left|A_{\gamma, \varepsilon}\right|^{2}\left|\chi_{\gamma, \varepsilon^{1 / 3}} \varphi_{\varepsilon}\right|^{2} \mathrm{~d} s \mathrm{~d} t \leqslant C \varepsilon^{14 / 3} a_{4}(\gamma, \varepsilon)^{2}\left\|w_{\varepsilon}\right\|_{L^{2}(\Omega)}^{2}, \\
& \int_{K(\gamma, \varepsilon)} \eta_{1, \tau(\varepsilon)}^{2}(t)\left|\left(\partial_{s}-\frac{i}{\varepsilon^{2}} A_{1}^{\varepsilon}\right)\left(\chi_{\gamma, \varepsilon^{1 / 3}} \varphi_{\varepsilon}\right)\right|^{2} \mathrm{~d} s \mathrm{~d} t \leqslant C \varepsilon^{-2} b_{0}(\gamma, \varepsilon)^{2}\left\|w_{\varepsilon}\right\|_{L^{2}(\Omega)}^{2} .
\end{aligned}
$$

Hence we have the following estimate for $q_{1}(\gamma, \varepsilon)$ :

$$
\left|q_{1}(\gamma, \varepsilon)\right| \leqslant C \varepsilon^{4 / 3} a_{4}(\gamma, \varepsilon) b_{0}(\gamma, \varepsilon)\left\|w_{\varepsilon}\right\|_{L^{2}(\Omega)}^{2} .
$$

Similarly, we obtain an estimate for $q_{2}(\gamma, \varepsilon)$ :

$$
\left|q_{2}(\gamma, \varepsilon)\right| \leqslant C \varepsilon^{8 / 3} a_{4}(\gamma, \varepsilon)^{2}\left\|w_{\varepsilon}\right\|_{L^{2}(\Omega)}^{2} .
$$

Combining (3.16) with (3.18)-(3.20), we get an estimate for $q(\gamma, \varepsilon)$ :

$$
|q(\gamma, \varepsilon)| \leqslant C \varepsilon^{4 / 3}\left[a_{4}(\gamma, \varepsilon) b_{0}(\gamma, \varepsilon)+\varepsilon^{4 / 3} a_{4}(\gamma, \varepsilon)^{2}\right]\left\|w_{\varepsilon}\right\|_{L^{2}(\Omega)}^{2} .
$$

Therefore

$$
\frac{2}{\varepsilon^{2}}|q(\varepsilon)| \leqslant C \varepsilon^{-2 / 3}\left\|w_{\varepsilon}\right\|_{L^{2}(\Omega)}^{2}
$$

Combining (3.15), (3.17) and (3.21), we get a lower bound for $Q_{\varepsilon^{2}, \mathbf{A}^{\varepsilon}}\left[w_{\varepsilon}\right]$ :

$$
Q_{\varepsilon^{2}, \mathbf{A}^{\varepsilon}}\left[w_{\varepsilon}\right] \geqslant \frac{1}{\varepsilon^{2}}\left(\beta_{0}-C_{1} \kappa_{\max } \varepsilon-C \varepsilon^{4 / 3}\right)\left\|w_{\varepsilon}\right\|_{L^{2}(\Omega)}^{2}
$$

Step 2. We estimate the second term in the right hand of (3.12).

Recall that $\eta_{1, \tau(\varepsilon)}=1$ if $t \leqslant \tau(\varepsilon) / 20$ and $\eta_{1, \tau(\varepsilon)}=0$ if $t \geqslant \tau(\varepsilon) / 10$. Hence we can find two positive constants $m_{1}<m_{2}$, both independent of $\varepsilon$, such that the support of $\left|\nabla \eta_{1, \tau(\varepsilon)}\right|$ is contained in a set $\left\{x \in \Omega: m_{1} \tau(\varepsilon) \leqslant t(x) \leqslant m_{2} \tau(\varepsilon)\right\}$ for all small $\varepsilon$. Thus

$$
\int_{\Omega}\left|\varphi_{\varepsilon} \nabla \eta_{1, \tau(\varepsilon)}\right|^{2} \mathrm{~d} x \leqslant \frac{c_{1}}{\tau(\varepsilon)^{2}} \int_{\left\{m_{1} \tau(\varepsilon) \leqslant t(x) \leqslant m_{2} \tau(\varepsilon)\right\}}\left|\varphi_{\varepsilon}\right|^{2} \mathrm{~d} x
$$




$$
\begin{aligned}
& \leqslant \frac{c_{2}}{\tau(\varepsilon)^{4}} \int_{\left\{m_{1} \tau(\varepsilon) \leqslant t(x) \leqslant m_{2} \tau(\varepsilon)\right\}} t(x)^{2}\left|\varphi_{\varepsilon}\right|^{2} \mathrm{~d} x \\
& \leqslant \frac{c_{3} \varepsilon^{2}}{\tau(\varepsilon)^{4}}\left\|\varphi_{\varepsilon}\right\|_{L^{2}(\Omega)}^{2} .
\end{aligned}
$$

Here we have used the first inequality in Lemma 3.3 with $k=2$.

Step 3. Now we can derive a lower bound for the eigenvalue $\mu_{\varepsilon}$.

As in step 2, we choose $\tau(\varepsilon)=\varepsilon^{5 / 12}$. From (3.10) we see that $\left\|w_{\varepsilon}\right\|_{L^{2}(\Omega)} \leqslant\left\|\varphi_{\varepsilon}\right\|_{L^{2}(\Omega)}$. Using (3.12), (3.22) and (3.23) we have

$$
\begin{aligned}
\mu_{\varepsilon}\left\|w_{\varepsilon}\right\|_{L^{2}(\Omega)}^{2} & =Q_{\varepsilon^{2}, \mathbf{A}^{\varepsilon}}\left[w_{\varepsilon}\right]-\int_{\Omega}\left|\varphi_{\varepsilon} \nabla \eta_{1, \tau(\varepsilon)}\right|^{2} \mathrm{~d} x \\
& \geqslant \frac{1}{\varepsilon^{2}}\left(\beta_{0}-C_{1} \kappa_{\max } \varepsilon-C \varepsilon^{4 / 3}\right)\left\|w_{\varepsilon}\right\|_{L^{2}(\Omega)}^{2}-\frac{C \varepsilon^{2}}{\tau(\varepsilon)^{4}}\left\|\varphi_{\varepsilon}\right\|_{L^{2}(\Omega)}^{2} \\
& \geqslant \frac{1}{\varepsilon^{2}}\left(\beta_{0}-C_{1} \kappa_{\max } \varepsilon-C \varepsilon^{4 / 3}-C \varepsilon^{7 / 3}\right)\left\|w_{\varepsilon}\right\|_{L^{2}(\Omega)}^{2},
\end{aligned}
$$

which yields a lower bound for $\mu_{\varepsilon}$

$$
\mu_{\varepsilon} \geqslant \frac{1}{\varepsilon^{2}}\left(\beta_{0}-C_{1} \kappa_{\max } \varepsilon-C \varepsilon^{4 / 3}\right)
$$

Now (3.2) is proved.

Remark 3.6. - (1) Lemmas 3.2-3.5 remain true under a weaker condition

$$
\begin{aligned}
& \left\|\mathbf{A}^{\varepsilon}\right\|_{C^{2}(\bar{\Omega})} \leqslant C, \\
& \left\|\operatorname{curl} \mathbf{A}^{\varepsilon}-1\right\|_{C^{1}(\bar{\Omega})}=\mathrm{o}(1), \quad \text { as } \varepsilon \rightarrow 0 .
\end{aligned}
$$

(2) We can show that, under condition (3.1), the eigenfunction $\varphi_{\varepsilon}$ decays exponentially inside the boundary and away from the set of the maximum points of the curvature. Since the proof is similar to the nonlinear case which will be discussed in Section 6, we omit it here.

From the proof above we see that the conclusion of [15] (Proposition 10.5) remains true.

PROPOSITION 3.7. - Under assumption (3.1), there exist $C>0$ and $\varepsilon_{0}>0$ such that, for any $0<\varepsilon<\varepsilon_{0}$ and $\phi \in W^{1,2}(\Omega)$, we have

$$
Q_{\varepsilon^{2}, \mathbf{A}^{\varepsilon}}[\phi] \geqslant \frac{1}{\varepsilon^{2}} \int_{\Omega} W_{\varepsilon}(x)|\phi|^{2} \mathrm{~d} x,
$$

where

$$
W_{\varepsilon}(x)= \begin{cases}1-C \varepsilon^{1 / 3} & \text { if } \operatorname{dist}(x, \partial \Omega)>2 \varepsilon^{1 / 3}, \\ \beta_{0}-C_{1} \kappa_{r}(s) \varepsilon-C \varepsilon^{4 / 3} & \text { if } \operatorname{dist}(x, \partial \Omega) \leqslant 2 \varepsilon^{1 / 3}\end{cases}
$$




\section{Elliptic estimates}

In this section we shall derive elliptic estimates for the minimal solutions of the Ginzburg-Landau system (2.2) when the applied field is close to $H_{C_{3}}$, namely when we assume

$$
\left(\frac{1}{\beta_{0}}+\mathrm{o}(1)\right) \kappa \leqslant \sigma<H_{C_{3}}(\kappa) .
$$

As before we use the notation: $\varepsilon=\frac{1}{\sqrt{\kappa \sigma}}$. Using conclusion (1) of Theorem A in Section 1, we see that condition (4.1) implies

$$
\varepsilon^{2} \kappa^{2}=\beta_{0}+\mathrm{o}(1), \quad \text { as } \varepsilon \rightarrow 0 .
$$

In the following, we denote by:

$\left(\psi^{\varepsilon}, \mathbf{A}^{\varepsilon}\right)$ : a non-trivial minimal solution of the Ginzburg-Landau system (2.2), $x^{\varepsilon}$ : a maximum point of $\left|\psi^{\varepsilon}(x)\right|$,

and we define $\lambda_{\varepsilon}$ by

$$
\lambda_{\varepsilon}=\left\|\psi^{\varepsilon}\right\|_{L^{\infty}(\Omega)}=\left|\psi^{\varepsilon}\left(x^{\varepsilon}\right)\right| .
$$

We always fix the gauge and assume that $\left(\psi^{\varepsilon}, A^{\varepsilon}\right)$ satisfies (2.3).

PROPOSITION 4.1. - Assume that condition (4.1) holds. Then we have:

$$
\operatorname{dist}\left(x^{\varepsilon}, \partial \Omega\right)=\mathrm{o}(\varepsilon) \quad \text { and } \quad\left\|\psi^{\varepsilon}\right\|_{L^{\infty}(\Omega)}=\mathrm{o}(1) \quad \text { as } \varepsilon \rightarrow 0 .
$$

For the proof, see [19] (Theorem 4.1 and the last paragraph of the proof of Lemma 4.5).

Proposition 4.2. - Under condition (4.1), there are positive constants $C$ and $\varepsilon_{0}$ such that, for all $0<\varepsilon \leqslant \varepsilon_{0}$, we have:

(1) $\left\|\nabla_{\frac{1}{\varepsilon^{2}} \mathbf{A}^{\varepsilon}} \psi^{\varepsilon}\right\|_{C(\bar{\Omega})} \leqslant \frac{C}{\varepsilon}\left\|\psi^{\varepsilon}\right\|_{L^{\infty}(\Omega)}$;

(2) $\left\|\operatorname{curl} \mathbf{A}^{\varepsilon}-1\right\|_{C^{1}(\bar{\Omega})} \leqslant C \varepsilon\left\|\psi^{\varepsilon}\right\|_{L^{\infty}(\Omega)}^{2}$;

(3) $\left\|\operatorname{curl} \mathbf{A}^{\varepsilon}-1\right\|_{C^{2}(\bar{\Omega})} \leqslant C\left\|\psi^{\varepsilon}\right\|_{L^{\infty}(\Omega)}^{2}$;

(4) For any multi-index $\alpha$ with $|\alpha| \leqslant 2$, there exist positive constants $c_{1}(\alpha), c_{2}(\alpha)$ such that, for all $0<\varepsilon<\varepsilon_{0}$, we have

$$
\left|D^{\alpha} \psi^{\varepsilon}(x)\right| \leqslant \frac{c_{1}(\alpha)}{\varepsilon^{|\alpha|}} \exp \left(-\frac{c_{2}(\alpha)}{\varepsilon} \operatorname{dist}(x, \partial \Omega)\right)\left\|\psi^{\varepsilon}\right\|_{L^{\infty}(\Omega)}, \quad \text { for all } x \in \Omega .
$$

Proof. - Step 1. We prove (1) by contradiction.

Choose $P^{\varepsilon} \in \bar{\Omega}$ such that

$$
\left|\nabla_{\frac{1}{\varepsilon^{2}} \mathbf{A}^{\varepsilon}} \psi^{\varepsilon}\left(P^{\varepsilon}\right)\right|=\left\|\nabla_{\frac{1}{\varepsilon^{2}} \mathbf{A}^{\varepsilon}} \psi^{\varepsilon}\right\|_{L^{\infty}(\Omega)} .
$$


Suppose conclusion (1) were false. Then there exists a sequence $\varepsilon_{j} \rightarrow 0$ such that

$$
\lim _{j \rightarrow \infty} \frac{\varepsilon_{j}}{\lambda_{\varepsilon_{j}}}\left\|\nabla_{\frac{1}{\varepsilon_{j}^{2}} \mathbf{A}^{\varepsilon_{j}}} \psi^{\varepsilon_{j}}\right\|_{L^{\infty}(\Omega)}=\infty .
$$

We shall find a contradiction. Passing to a subsequence if necessary, we deal with two cases, namely, case 1 where $P^{\varepsilon_{j}}$ stays away from the boundary for all $j$, and case 2 where $P^{\varepsilon_{j}}$ is close to the boundary as $j \rightarrow \infty$. In the following, for the simplicity of notations, we denote $\varepsilon_{j}$ by $\varepsilon$, and consider a "sequence" $\{\varepsilon\}$.

Case $1 . \lim _{\varepsilon \rightarrow 0} \frac{1}{\varepsilon} \operatorname{dist}\left(P^{\varepsilon}, \partial \Omega\right)=\infty$.

We shall show that, in this case, as $\varepsilon \rightarrow 0$,

$$
\left\|\nabla_{\frac{1}{\varepsilon^{2}} \mathbf{A}^{\varepsilon}} \psi^{\varepsilon}\right\|_{L^{\infty}(\Omega)}=\mathrm{o}\left(\frac{\lambda_{\varepsilon}}{\varepsilon}\right) .
$$

Define the rescaled functions as follows:

$$
\begin{aligned}
& \mathbf{A}_{\varepsilon}(y)=\frac{1}{\varepsilon}\left[\mathbf{A}^{\varepsilon}\left(P^{\varepsilon}+\varepsilon y\right)-\mathbf{A}^{\varepsilon}\left(P^{\varepsilon}\right)\right], \\
& \phi_{\varepsilon}(y)=\frac{1}{\lambda_{\varepsilon}} \exp \left(-\frac{i}{\varepsilon} \mathbf{A}^{\varepsilon}\left(P^{\varepsilon}\right) \cdot y\right) \psi^{\varepsilon}\left(P^{\varepsilon}+\varepsilon y\right) .
\end{aligned}
$$

From (2.2), $\phi_{\varepsilon}$ satisfies

$$
\left\{\begin{array}{l}
-\nabla_{\mathbf{A}_{\varepsilon}}^{2} \phi_{\varepsilon}=\varepsilon^{2} \kappa^{2}\left(1-\lambda_{\varepsilon}^{2}\left|\phi_{\varepsilon}\right|^{2}\right) \phi_{\varepsilon}, \\
\operatorname{curl}^{2} \mathbf{A}_{\varepsilon}=\varepsilon^{2} \lambda_{\varepsilon}^{2} \Im\left(\overline{\phi_{\varepsilon}} \nabla_{\mathbf{A}_{\varepsilon}} \phi_{\varepsilon}\right), \quad \text { in }\left(\Omega-P^{\varepsilon}\right) / \varepsilon .
\end{array}\right.
$$

Recall that $\varepsilon^{2} \kappa^{2} \rightarrow \beta_{0}<1$ as $\varepsilon \rightarrow 0$. Using (4.2) and applying the argument in [19] (Lemma 4.2), we can show that, for any $R>0$, there exists $\varepsilon_{0}(R)>0$ such that, for $0<\varepsilon<\varepsilon_{0}(R)$,

$$
\left\|\mathbf{A}_{\varepsilon}\right\|_{C^{2, \alpha}\left(B_{R}\right)} \leqslant C(R) .
$$

We also obtain (see [19], Lemma 4.4): $\phi_{\varepsilon} \rightarrow 0$ in $C_{\text {loc }}^{2, \alpha}\left(\mathbb{R}^{2}\right.$ ) as $\varepsilon \rightarrow 0$. Therefore,

$$
\nabla_{\mathbf{A}_{\varepsilon}} \phi_{\varepsilon} \rightarrow 0 \quad \text { in } C_{\mathrm{loc}}^{1, \alpha}\left(\mathbb{R}^{2}\right) \text {, as } \varepsilon \rightarrow 0 \text {. }
$$

Here we mean by convergence in $C_{\text {loc }}^{k, \alpha}$, that for any compact subset $K \subset \mathbb{R}^{2}$, there exists $\varepsilon_{K}>0$ such that we have convergence of $\phi_{\varepsilon}$ (respectively $\nabla_{\mathbf{A}_{\varepsilon}} \phi_{\varepsilon}$ ) in $C^{k, \alpha}(K)$ as $\varepsilon \rightarrow 0$ $\left(0<\varepsilon<\varepsilon_{K}\right)$. Hence, as $\varepsilon \rightarrow 0$,

$$
\left\|\nabla_{\frac{1}{\varepsilon^{2}} \mathbf{A}^{\varepsilon}} \psi^{\varepsilon}\right\|_{L^{\infty}(\Omega)}=\left|\nabla_{\frac{1}{\varepsilon^{2}} \mathbf{A}^{\varepsilon}} \psi^{\varepsilon}\left(P^{\varepsilon}\right)\right|=\frac{\lambda_{\varepsilon}}{\varepsilon}\left|\nabla_{\mathbf{A}_{\varepsilon}} \phi_{\varepsilon}(0)\right|=\mathrm{o}\left(\frac{\lambda_{\varepsilon}}{\varepsilon}\right) .
$$

So (4.4) holds.

Case 2. $\operatorname{dist}\left(P^{\varepsilon}, \partial \Omega\right) \leqslant C \varepsilon$ as $\varepsilon \rightarrow 0$.

We shall show that, in this case, as $\varepsilon \rightarrow 0$, we have

$$
\left\|\nabla_{\frac{1}{\varepsilon^{2}} \mathbf{A}^{\varepsilon}} \psi^{\varepsilon}\right\|_{L^{\infty}(\Omega)}=\mathrm{O}\left(\frac{\lambda_{\varepsilon}}{\varepsilon}\right) .
$$


Choose $Q^{\varepsilon} \in \partial \Omega$ such that $\left|P^{\varepsilon}-Q^{\varepsilon}\right|=\operatorname{dist}\left(P^{\varepsilon}, \partial \Omega\right)$. Let $\mathcal{F}_{\varepsilon}$ be the diffeomorphism which straightens a portion of the boundary around $Q^{\varepsilon}$ as defined in [19] (Section 2) with $\mathcal{F}_{\varepsilon}(0)=Q^{\varepsilon}$. Let us define $y^{\varepsilon}=\mathcal{F}_{\varepsilon}^{-1}\left(x^{\varepsilon}\right)$ and $z^{\varepsilon}=y^{\varepsilon} / \varepsilon$. Note that $\left\{z^{\varepsilon}\right\}$ is bounded as $\varepsilon \rightarrow 0$. Without loss of generality, we may assume $\lim _{\varepsilon \rightarrow 0} z^{\varepsilon}=z^{0}$. Note that, although $\mathcal{F}_{\varepsilon}$ depends on $\varepsilon$, its domain contains a ball $R_{R_{0}}$ with $R_{0}$ independent of $\varepsilon$, and both $\mathcal{F}_{\varepsilon}$ and det $D \mathcal{F}_{\varepsilon}$ are uniformly smooth on this ball. For simplicity, we write now $\mathcal{F}=\mathcal{F}_{\varepsilon}$ and $g=\operatorname{det} D \mathcal{F}_{\varepsilon}$. Let $\tilde{\psi}^{\varepsilon}(y)=\psi^{\varepsilon}(\mathcal{F}(y))$ and let

$$
\mathbf{a}^{\varepsilon}=\left[g \mathbf{A}^{\varepsilon} \cdot \mathbf{e}_{1}\right] \mathbf{e}_{1}+\left[\mathbf{A}^{\varepsilon} \cdot \mathbf{e}_{2}\right] \mathbf{e}_{2}
$$

be the vector field associated with $\mathbf{A}^{\varepsilon}$, see [19] ((2.22) and (2.23)). Note that $\mathbf{a}^{\varepsilon}(0)=$ $\mathbf{A}^{\varepsilon}\left(Q^{\varepsilon}\right), \mathbf{a}^{\varepsilon}(0) \cdot \mathbf{e}_{2}=0$. Next we define the following rescaled functions

$$
\begin{aligned}
& \chi_{\varepsilon}=\frac{1}{\varepsilon} y_{1} \mathbf{A}^{\varepsilon}\left(Q^{\varepsilon}\right) \cdot \mathbf{e}_{1}, \\
& \tilde{\phi}_{\varepsilon}(y)=\frac{1}{\lambda_{\varepsilon}} \exp \left(-i \chi_{\varepsilon}\right) \tilde{\psi}^{\varepsilon}(\varepsilon y), \\
& \mathbf{a}_{\varepsilon}(y)=\frac{1}{\varepsilon}\left[\mathbf{a}^{\varepsilon}(\varepsilon y)-\mathbf{a}^{\varepsilon}(0)\right], \\
& g_{\varepsilon}(y)=g(\varepsilon y) .
\end{aligned}
$$

Using (2.2) and the gauge invariance property, we get the equation for $\tilde{\phi}_{\varepsilon}$ :

$$
\begin{cases}-\Delta\left(g_{\varepsilon}\right)_{\mathbf{a}_{\varepsilon}} \tilde{\phi}_{\varepsilon}=\varepsilon^{2} \kappa^{2}\left(1-\lambda_{\varepsilon}^{2}\left|\tilde{\phi}_{\varepsilon}\right|^{2}\right) \tilde{\phi}_{\varepsilon} & \text { in } B_{R_{0} / \varepsilon}^{+}, \\ \partial_{2} \tilde{\phi}_{\varepsilon}=0 & \text { on } \Gamma_{R_{0} / \varepsilon} .\end{cases}
$$

As in [19] (Section 4), we use (4.2) to show that, there is a subsequence (still denoted by $\tilde{\phi}_{\varepsilon}$ ) such that $\tilde{\phi}_{\varepsilon} \rightarrow \tilde{\phi}$ and $\mathbf{a}_{\varepsilon} \rightarrow \omega(y)$ in $C_{\text {loc }}^{2+\alpha}\left(\mathbb{R}_{+}^{2}\right)$ as $\varepsilon \rightarrow 0$, and $\tilde{\phi}$ is a bounded solution of (2.6). Therefore, as $\varepsilon \rightarrow 0$,

$$
D\left(g_{\varepsilon}\right)_{\mathbf{a}_{\varepsilon}} \tilde{\phi}_{\varepsilon} \rightarrow \nabla_{\omega} \tilde{\phi} \quad \text { in } C_{\mathrm{loc}}^{1+\alpha}\left(\mathbb{R}_{+}^{2}\right) .
$$

Especially, $D\left(g_{\varepsilon}\right)_{\mathbf{a}_{\varepsilon}} \tilde{\phi}_{\varepsilon}(0) \rightarrow \nabla_{\omega} \tilde{\phi}(0)$, so $\nabla_{\mathbf{A}_{\varepsilon}} \phi_{\varepsilon}(0) \rightarrow \nabla_{\omega} \tilde{\phi}(0)$. Hence, as $\varepsilon \rightarrow 0$,

$$
\left\|\nabla_{\frac{1}{\varepsilon^{2}} \mathbf{A}^{\varepsilon}} \psi^{\varepsilon}\right\|_{L^{\infty}(\Omega)}=\left|\nabla_{\frac{1}{\varepsilon^{2}} \mathbf{A}^{\varepsilon}} \psi^{\varepsilon}\left(P^{\varepsilon}\right)\right|=\frac{\lambda_{\varepsilon}}{\varepsilon}\left|\nabla_{\mathbf{A}_{\varepsilon}} \phi_{\varepsilon}(0)\right|=\mathrm{O}\left(\frac{\lambda_{\varepsilon}}{\varepsilon}\right) .
$$

So (4.6) is true.

Combining (4.4) and (4.6) we get a contradiction to (4.3). So conclusion (1) is proved. Step 2. We prove (2).

From the second equation of (2.2) and using conclusion (1) we have:

$$
\begin{aligned}
\left\|\nabla\left[\operatorname{curl}\left(\mathbf{A}^{\varepsilon}-\mathbf{F}\right)\right]\right\|_{L^{\infty}(\Omega)} & =\left\|\operatorname{curl}^{2}\left(\mathbf{A}^{\varepsilon}-\mathbf{F}\right)\right\|_{L^{\infty}(\Omega)} \\
& =\varepsilon^{2}\left\|\Im\left(\overline{\psi^{\varepsilon}} \nabla_{\frac{1}{\varepsilon^{2}} \mathbf{A}^{\varepsilon}} \psi^{\varepsilon}\right)\right\|_{L^{\infty}(\Omega)} \\
& \leqslant \varepsilon^{2}\left\|\psi^{\varepsilon}\right\|_{L^{\infty}(\Omega)}\left\|\nabla_{\frac{1}{\varepsilon^{2}} \mathbf{2}^{\varepsilon}} \psi^{\varepsilon}\right\|_{L^{\infty}(\Omega)}
\end{aligned}
$$




$$
\leqslant \varepsilon^{2}\left\|\psi^{\varepsilon}\right\|_{L^{\infty}(\Omega)} \cdot \frac{C}{\varepsilon}\left\|\psi^{\varepsilon}\right\|_{L^{\infty}(\Omega)}=C \varepsilon\left\|\psi^{\varepsilon}\right\|_{L^{\infty}(\Omega)}^{2} .
$$

Recall that $\operatorname{curl}\left(\mathbf{A}^{\varepsilon}-\mathbf{F}\right)=0$ on $\partial \Omega$. The above inequalities imply that

$$
\left\|\operatorname{curl}\left(\mathbf{A}^{\varepsilon}-\mathbf{F}\right)\right\|_{L^{\infty}(\Omega)} \leqslant C^{\prime}\left\|\nabla\left[\operatorname{curl}\left(\mathbf{A}^{\varepsilon}-\mathbf{F}\right)\right]\right\|_{L^{\infty}(\Omega)} \leqslant C C^{\prime} \varepsilon\left\|\psi^{\varepsilon}\right\|_{L^{\infty}(\Omega)}^{2} .
$$

Therefore

$$
\left\|\operatorname{curl}\left(\mathbf{A}^{\varepsilon}-\mathbf{F}\right)\right\|_{C^{1}(\bar{\Omega})} \leqslant C^{\prime \prime} \varepsilon\left\|\psi^{\varepsilon}\right\|_{L^{\infty}(\Omega)}^{2} .
$$

Hence conclusion (2) holds (replacing $C$ by a larger number).

Step 3. We prove (3).

CLAIM 1. - Under condition (4.1), there exist positive constants $M$ and $\varepsilon_{0}$ such that, for all $0<\varepsilon<\varepsilon_{0}$, we have

$$
\left\|\nabla\left(\overline{\psi^{\varepsilon}} \nabla_{\frac{1}{\varepsilon^{2}} \mathbf{A}^{\varepsilon}} \psi^{\varepsilon}\right)\right\|_{L^{\infty}(\Omega)} \leqslant \frac{C}{\varepsilon^{2}}\left\|\psi^{\varepsilon}\right\|_{L^{\infty}(\Omega)}^{2} .
$$

The proof of Claim 1 is similar to the proof of conclusion (1). Choose $\hat{P}^{\varepsilon} \in \bar{\Omega}$ such that

$$
\left|\left[\nabla\left(\overline{\psi^{\varepsilon}} \nabla_{\frac{1}{\varepsilon^{2}} \mathbf{A}^{\varepsilon}} \psi^{\varepsilon}\right)\right]\left(\hat{P}^{\varepsilon}\right)\right|=\left\|\nabla\left(\overline{\psi^{\varepsilon}} \nabla_{\frac{1}{\varepsilon^{2}} \mathbf{A}^{\varepsilon}} \psi^{\varepsilon}\right)\right\|_{L^{\infty}(\Omega)} .
$$

Define the rescaled functions as follows:

$$
\begin{aligned}
& \hat{\mathbf{A}}_{\varepsilon}(y)=\frac{1}{\varepsilon}\left[\mathbf{A}^{\varepsilon}\left(\hat{P}^{\varepsilon}+\varepsilon y\right)-\mathbf{A}^{\varepsilon}\left(\hat{P}^{\varepsilon}\right)\right], \\
& \hat{\phi}_{\varepsilon}(y)=\frac{1}{\lambda_{\varepsilon}} \exp \left(-\frac{i}{\varepsilon} \mathbf{A}^{\varepsilon}\left(\hat{P}^{\varepsilon}\right) \cdot y\right) \psi^{\varepsilon}\left(\hat{P}^{\varepsilon}+\varepsilon y\right) .
\end{aligned}
$$

$\hat{\phi}_{\varepsilon}$ satisfies an equation similar to $(4.5)$ in $\Omega(\varepsilon)=\left(\Omega-\hat{P}^{\varepsilon}\right) / \varepsilon$. As in step 1 we can show that, there exist $M>0$ and $\varepsilon_{0}>0$ such that, for any $0<\varepsilon<\varepsilon_{0}$, we have

$$
\left\|\nabla\left(\overline{\hat{\phi}}_{\varepsilon} \nabla_{\frac{1}{\varepsilon^{2}} \hat{\mathbf{A}}_{\varepsilon}} \hat{\phi}_{\varepsilon}\right)\right\|_{L^{\infty}(\Omega(\varepsilon))} \leqslant M
$$

We compute

$$
\begin{aligned}
& \psi^{\varepsilon}\left(\hat{P}^{\varepsilon}+\varepsilon y\right)=\lambda_{\varepsilon} \hat{\phi}_{\varepsilon}(y) \exp \left(\frac{i}{\varepsilon} \mathbf{A}^{\varepsilon}\left(\hat{P}^{\varepsilon}\right) \cdot y\right), \\
& \left(\nabla_{\frac{1}{\varepsilon^{2}}} \mathbf{A}^{\varepsilon} \psi^{\varepsilon}\right)\left(\hat{P}^{\varepsilon}+\varepsilon y\right)=\frac{\lambda_{\varepsilon}}{\varepsilon} \exp \left(\frac{i}{\varepsilon} \mathbf{A}^{\varepsilon}\left(\hat{P}^{\varepsilon}\right) \cdot y\right)\left(\nabla_{\hat{\mathbf{A}}_{\varepsilon}} \hat{\phi}_{\varepsilon}\right)(y), \\
& \left(\overline{\psi^{\varepsilon}} \nabla_{\frac{1}{\varepsilon^{2}} \mathbf{A}^{\varepsilon}} \psi^{\varepsilon}\right)\left(\hat{P}^{\varepsilon}+\varepsilon y\right)=\frac{\lambda_{\varepsilon}^{2}}{\varepsilon}\left(\overline{\hat{\phi}}_{\varepsilon} \nabla_{\hat{\mathbf{A}}_{\varepsilon}} \hat{\phi}_{\varepsilon}\right)(y), \\
& \frac{\partial}{\partial x_{j}}\left(\overline{\psi^{\varepsilon}} \nabla_{\frac{1}{\varepsilon^{2}} \mathbf{A}^{\varepsilon}} \psi^{\varepsilon}\right)\left(\hat{P}^{\varepsilon}+\varepsilon y\right)=\frac{\lambda_{\varepsilon}^{2}}{\varepsilon^{2}} \frac{\partial}{\partial y_{j}}\left(\overline{\hat{\phi}_{\varepsilon}} \nabla_{\hat{\mathbf{A}}_{\varepsilon}} \hat{\phi}_{\varepsilon}\right)(y) .
\end{aligned}
$$




$$
\left.\left\|\nabla\left(\overline{\psi^{\varepsilon}} \nabla_{\frac{1}{\varepsilon^{2}}{ }^{\varepsilon}} \psi^{\varepsilon}\right)\right\|_{L^{\infty}(\Omega)}=\mid\left[\nabla\left(\overline{\psi^{\varepsilon}} \nabla_{\frac{1}{\varepsilon^{2}} \mathbf{A}^{\varepsilon}} \psi^{\varepsilon}\right)\right]\left(\hat{P}^{\varepsilon}\right)\right]\left|=\frac{\lambda_{\varepsilon}^{2}}{\varepsilon^{2}}\right| \nabla\left(\overline{\hat{\phi}}_{\varepsilon} \nabla_{\hat{\mathbf{A}}_{\varepsilon}} \hat{\phi}_{\varepsilon}\right)(y) \mid \leqslant \frac{M \lambda_{\varepsilon}^{2}}{\varepsilon^{2}} .
$$

Claim 1 follows.

Note that

$$
\operatorname{curl}^{2}\left(\mathbf{A}^{\varepsilon}-\mathbf{F}\right)=\left(\partial_{2}\left[\operatorname{curl}\left(\mathbf{A}^{\varepsilon}-\mathbf{F}\right)\right],-\partial_{1}\left[\operatorname{curl}\left(\mathbf{A}^{\varepsilon}-\mathbf{F}\right)\right]\right) .
$$

Hence, using Claim 1 and the second equation of (2.2), we have

$$
\begin{aligned}
\left\|\partial_{i j}\left[\operatorname{curl}\left(\mathbf{A}^{\varepsilon}-\mathbf{F}\right)\right]\right\|_{L^{\infty}(\Omega)} & \leqslant\left\|\partial_{j}\left[\operatorname{curl}^{2}\left(\mathbf{A}^{\varepsilon}-\mathbf{F}\right)\right]\right\|_{L^{\infty}(\Omega)} \\
& =\varepsilon^{2}\left\|\partial_{j} \Im\left(\overline{\psi^{\varepsilon}} \nabla_{\frac{1}{\varepsilon^{2}} \mathbf{A}^{\varepsilon}} \psi^{\varepsilon}\right)\right\|_{L^{\infty}(\Omega)} \\
& =\varepsilon^{2}\left\|\Im \partial_{j}\left(\overline{\psi^{\varepsilon}} \nabla_{\frac{1}{\varepsilon^{2}} \mathbf{A}^{\varepsilon}} \psi^{\varepsilon}\right)\right\|_{L^{\infty}(\Omega)} \\
& \leqslant \varepsilon^{2}\left\|\partial_{j}\left(\overline{\psi^{\varepsilon}} \nabla_{\frac{1}{\varepsilon^{2}} \mathbf{A}^{\varepsilon}} \psi^{\varepsilon}\right)\right\|_{L^{\infty}(\Omega)} \leqslant M \lambda_{\varepsilon}^{2} .
\end{aligned}
$$

Combining this and conclusion (2) we obtain conclusion (3).

Step 4. Conclusion (4) can be proved as in [9] (Theorem 4.3) and [15] (Theorem 6.3). In fact, conclusion (2) implies that, as $\varepsilon \rightarrow 0,\left\|\operatorname{curl} \mathbf{A}^{\varepsilon}-1\right\|_{C(\bar{\Omega})}=\mathrm{o}(1)$. Then applying the arguments in [9] or [15] to the equation for $\psi^{\varepsilon}$ one obtains conclusion (4).

Now, Proposition 4.2 is completed.

Remark 4.3. - (1) From step 1 of the proof of Proposition 4.2 we see that, for any sequence $\rho_{\varepsilon} \rightarrow \infty$,

$$
\lim _{\varepsilon \rightarrow 0} \max _{\operatorname{dist}(x, \partial \Omega) \geqslant \varepsilon \rho_{\varepsilon}} \frac{\varepsilon}{\lambda_{\varepsilon}}\left|\nabla_{\frac{1}{\varepsilon^{2}} \mathbf{A}^{\varepsilon}} \psi^{\varepsilon}(x)\right|=0 .
$$

(2) Inequality (4.7) says that $\left|\nabla\left(\overline{\psi^{\varepsilon}} \nabla_{\frac{1}{\varepsilon^{2}} \mathbf{A}^{\varepsilon}} \psi^{\varepsilon}\right)\right|$ is of order $\mathrm{O}\left(\lambda_{\varepsilon}^{2} / \varepsilon^{2}\right)$. However, we can not conclude that $\left|\nabla\left(\nabla_{\frac{1}{2}} \mathbf{A}^{\varepsilon} \psi^{\varepsilon}\right)\right|$ is also of order $\mathrm{O}\left(\lambda_{\varepsilon} / \varepsilon^{2}\right)$. In fact, from the computations in step 3 of the proof of Proposition 4.2 we see that

$$
\begin{aligned}
{\left[\partial_{j}\left(\nabla_{\frac{1}{\varepsilon^{2}} \mathbf{A}^{\varepsilon}} \psi^{\varepsilon}\right)\right]\left(\hat{P}^{\varepsilon}+\varepsilon y\right)=} & \frac{i \lambda_{\varepsilon}}{\varepsilon^{3}} \mathbf{A}^{\varepsilon}\left(\hat{P}^{\varepsilon}\right) \exp \left(\frac{i}{\varepsilon} \mathbf{A}^{\varepsilon}\left(\hat{P}^{\varepsilon}\right) \cdot y\right)\left(\nabla_{\hat{\mathbf{A}}_{\varepsilon}} \hat{\phi}_{\varepsilon}\right)(y) \\
& +\frac{\lambda_{\varepsilon}}{\varepsilon^{2}} \exp \left(\frac{i}{\varepsilon} \mathbf{A}^{\varepsilon}\left(\hat{P}^{\varepsilon}\right) \cdot y\right)\left[\partial_{j}\left(\nabla_{\hat{\mathbf{A}}_{\varepsilon}} \hat{\phi}_{\varepsilon}\right)\right](y) .
\end{aligned}
$$

Thus, as $\varepsilon \rightarrow 0$, we have:

$$
\left\|\nabla\left(\nabla_{\frac{1}{\varepsilon^{2}} \mathbf{A}^{\varepsilon}} \psi^{\varepsilon}\right)\right\|_{L^{\infty}(\Omega)}=\mathrm{O}\left(\frac{\lambda_{\varepsilon}}{\varepsilon^{3}}\right) .
$$

The following theorem will be used in Section 5 to establish the lower bound of $H_{C_{3}}(\kappa)$.

THEOREM 4.4. - Assume that condition (4.1) holds and that $\left(\psi^{\varepsilon}, \mathbf{A}^{\varepsilon}\right)$ is a minimal solution of (2.2). Then there exist $C>0$ and $\varepsilon_{0}>0$ such that, for all $0<\varepsilon<\varepsilon_{0}$, the lowest eigenvalue $\mu\left(\frac{1}{\varepsilon^{2}} \mathbf{A}^{\varepsilon}\right)$ of the Neumann-Schrödinger operator $-\nabla_{\frac{1}{\varepsilon^{2}}}^{2} \mathbf{A}^{\varepsilon}$ satisfies the 
following inequality:

$$
\frac{1}{\varepsilon^{2}}\left(\beta_{0}-C_{1} \kappa_{\max } \varepsilon-C \varepsilon^{4 / 3}\right) \leqslant \mu\left(\frac{1}{\varepsilon^{2}} \mathbf{A}^{\varepsilon}\right) \leqslant \kappa^{2} .
$$

Here $C_{1}$ is the number given in (2.7).

Proof. - Since $\sigma<H_{C_{3}}(\kappa),(2.2)$ has a non-trivial minimal solution $\left(\psi^{\varepsilon}, \mathbf{A}^{\varepsilon}\right)$. From Lemma 2.1(2) we have

$$
\mu\left(\frac{1}{\varepsilon^{2}} \mathbf{A}^{\varepsilon}\right)<\kappa^{2}
$$

for all $\varepsilon>0$.

From Proposition 4.2 we see that the family $\left\{\mathbf{A}^{\varepsilon}\right\}_{0<\varepsilon<\varepsilon_{0}}$ satisfies condition (3.1). Using Theorem 3.1 we conclude that, the lowest eigenvalue $\mu_{\varepsilon}=\mu\left(\frac{1}{\varepsilon^{2}} \mathbf{A}^{\varepsilon}\right)$ satisfies

$$
\mu\left(\frac{1}{\varepsilon^{2}} \mathbf{A}^{\varepsilon}\right) \geqslant \frac{1}{\varepsilon^{2}}\left(\beta_{0}-C_{1} \kappa_{\max } \varepsilon-C \varepsilon^{4 / 3}\right)
$$

for all $0<\varepsilon<\varepsilon_{0}$. Hence (4.8) holds.

Next we give $L^{2}$ estimates for $\left|\psi^{\varepsilon}\right|$, which will be used in Section 6. The conclusions indicate that, if the applied field is close to the upper critical field in the sense of condition (4.1), then the order parameter concentrates in a thin layer around the boundary $\partial \Omega$.

LEMMA 4.5. - Assume that condition (4.1) holds and $\mathbf{F}$ satisfies (1.3). Let $\left(\psi^{\varepsilon}, \mathbf{A}^{\varepsilon}\right)$ be a minimal solution of (2.2). There exist positive constants $M, c$ and $\varepsilon_{0}$ such that, for all $0<\varepsilon \leqslant \varepsilon_{0}$, we have

$$
\int_{\Omega}\left|\psi^{\varepsilon}\right|^{2} \mathrm{~d} x \leqslant M \int_{\{\operatorname{dist}(x, \partial \Omega) \leqslant c \varepsilon\}}\left|\psi^{\varepsilon}\right|^{2} \mathrm{~d} x .
$$

Proof. - Note that the weighted $L^{2}$ estimate (3.4) is valid for $\psi^{\varepsilon}$. In fact, from (2.2) we have, for any real and smooth function $\chi$,

$$
\begin{aligned}
\int_{\Omega}\left|\nabla_{\frac{1}{\varepsilon^{2}} \boldsymbol{A}^{\varepsilon}}\left(\chi \psi^{\varepsilon}\right)\right|^{2} \mathrm{~d} x & =\int_{\Omega}\left\{\kappa^{2}\left(1-\left|\psi^{\varepsilon}\right|^{2}\right)\left|\chi \psi^{\varepsilon}\right|^{2}+\left|\psi^{\varepsilon} \nabla \chi\right|^{2}\right\} \mathrm{d} x \\
& \leqslant \int_{\Omega}\left\{\kappa^{2}\left|\chi \psi^{\varepsilon}\right|^{2}+\left|\psi^{\varepsilon} \nabla \chi\right|^{2}\right\} \mathrm{d} x .
\end{aligned}
$$

On the other hand, using Proposition 4.2 (conclusion (2)) we see that, if $\chi$ is supported in the interior of $\Omega$, then

$$
\int_{\Omega}\left|\nabla_{\frac{1}{\varepsilon^{2}} \mathbf{A}^{\varepsilon}}\left(\chi \psi^{\varepsilon}\right)\right|^{2} \mathrm{~d} x \geqslant \inf _{x \in \Omega}\left|\operatorname{curl} \mathbf{A}^{\varepsilon}\right|^{2} \int_{\Omega}\left|\chi \psi^{\varepsilon}\right|^{2} \mathrm{~d} x \geqslant(1-C \varepsilon) \int_{\Omega}\left|\chi \psi^{\varepsilon}\right|^{2} \mathrm{~d} x .
$$


From these inequalities, we can argue as in [15] (Section 6.4) to get

$$
\int_{\Omega} \exp \left(\frac{\alpha t(x)}{\varepsilon}\right)\left|\psi^{\varepsilon}\right|^{2} \mathrm{~d} x \leqslant C \int_{\Omega}\left|\psi^{\varepsilon}\right|^{2} \mathrm{~d} x,
$$

where $\alpha$ and $C$ are independent of $\varepsilon$. Then, using the above inequalities we can show that (see [15], (6.26))

$$
\int_{\Omega} \exp \left(\frac{\alpha t(x)}{\varepsilon}\right)\left|\nabla_{\frac{1}{\varepsilon^{2}} \mathbf{A}^{\varepsilon}} \psi^{\varepsilon}\right|^{2} \mathrm{~d} x \leqslant \frac{C}{\varepsilon^{2}} \int_{\Omega}\left|\psi^{\varepsilon}\right|^{2} \mathrm{~d} x .
$$

Thus the weighted $L^{2}$ estimates are valid for $\psi^{\varepsilon}$.

Especially we have

$$
\int_{\{\operatorname{dist}(x, \partial \Omega) \geqslant c \varepsilon\}}\left|\psi^{\varepsilon}\right|^{2} \mathrm{~d} x \leqslant C \mathrm{e}^{-\alpha c} \int_{\Omega}\left|\psi^{\varepsilon}\right|^{2} \mathrm{~d} x .
$$

Hence

$$
\left(1-C \mathrm{e}^{-\alpha c}\right) \int_{\{\operatorname{dist}(x, \partial \Omega) \geqslant c \varepsilon\}}\left|\psi^{\varepsilon}\right|^{2} \mathrm{~d} x \leqslant C \mathrm{e}^{-\alpha c} \int_{\{\operatorname{dist}(x, \partial \Omega)<c \varepsilon\}}\left|\psi^{\varepsilon}\right|^{2} \mathrm{~d} x .
$$

Choose $c>0$ such that $1-C \mathrm{e}^{-\alpha c} \geqslant 1 / 2$. From the above inequality we find:

$$
\int_{\Omega}\left|\psi^{\varepsilon}\right|^{2} \mathrm{~d} x \leqslant \frac{1}{\left(1-C \mathrm{e}^{-\alpha c}\right)} \int_{\{\operatorname{dist}(x, \partial \Omega)<c \varepsilon\}}\left|\psi^{\varepsilon}\right|^{2} \mathrm{~d} x .
$$

So (4.9) holds with $M=1 /\left(1-C \mathrm{e}^{-\alpha c}\right)$.

\section{Upper bound estimates for $H_{C_{3}}(\kappa)$}

In this section we keep the notations of Section 1.

Proposition 5.1. - Assume that $\Omega$ is a bounded simply-connected 2-dimensional domain of class $C^{4}$. Then we have, for $\kappa$ large,

$$
H_{C_{3}}(\kappa) \leqslant \frac{\kappa}{\beta_{0}}+\frac{C_{1}}{\beta_{0}^{3 / 2}} \kappa_{\max }+\mathrm{O}\left(\kappa^{-1 / 3}\right) .
$$

Proof. - We shall prove the following conclusion: For any $\sigma$ satisfying $0<\sigma<$ $H_{C_{3}}(\kappa)$, we have

$$
\sigma<\frac{\kappa}{\beta_{0}}+\frac{C_{1}}{\beta_{0}^{3 / 2}} \kappa_{\max }+\mathrm{O}\left(\kappa^{-1 / 3}\right) .
$$

(5.1) follows from this conclusion. 
From the lower bound of $H_{C_{3}}$, we only need to consider such $\sigma$ 's that

$$
\frac{\kappa}{\beta_{0}}+\frac{C_{1}}{\beta_{0}^{3 / 2}} \kappa_{\max }-\frac{L}{\kappa^{1 / 3}}<\sigma<H_{C_{3}}(\kappa)
$$

with $L>0$ large. For $\sigma$ satisfying (5.3), condition (4.1) holds. Ginzburg-Landau system (2.2) has a non-trivial minimal solution $\left(\psi^{\varepsilon}, \mathbf{A}^{\varepsilon}\right)$. From Theorem 4.4 , for all $0<\varepsilon<\varepsilon_{0}$, the lowest eigenvalue $\mu_{\varepsilon}=\mu\left(\frac{1}{\varepsilon^{2}} \mathbf{A}^{\varepsilon}\right)$ satisfies (4.8). From (4.8) and (5.3) we have

$$
\frac{\kappa}{\sigma}=\varepsilon^{2} \kappa^{2}>\beta_{0}-C_{1} \kappa_{\max } \varepsilon-C \varepsilon^{4 / 3} .
$$

Inequality (5.4) implies that

$$
\frac{1}{\sigma}>\frac{\beta_{0}}{\kappa}-\frac{C_{1}}{\sigma^{1 / 2} \kappa^{3 / 2}} \kappa_{\max }-\frac{C}{\sigma^{2 / 3} \kappa^{5 / 3}}
$$

and

$$
\frac{1}{\sigma}=\frac{\beta}{\kappa}-\mathrm{O}\left(\frac{1}{\kappa^{3 / 2}}\right)
$$

Plugging (5.6) into the right side of (5.5), we get

$$
\frac{1}{\sigma}>\frac{\beta_{0}}{\kappa}-\frac{C_{1} \sqrt{\beta_{0}}}{\kappa^{2}} \kappa_{\max }-\mathrm{O}\left(\frac{1}{\kappa^{7 / 3}}\right) .
$$

Thus $\sigma$ must satisfy (5.2). So (5.1) holds.

Proof of Theorem 1.1. - Theorem 1.1 follows from Propositions 2.3 and 5.1.

\section{Localization of order parameters}

In this section we assume that $\Omega$ is a bounded simply-connected 2-dimensional domain of class $C^{4}$ and discuss the concentration phenomena of order parameters when the applied field is close to $H_{C_{3}}(\kappa)$ and $\kappa$ is large. We keep the notation $\varepsilon=1 / \sqrt{\sigma \kappa}$ and denote by $\left(\psi^{\varepsilon}, \mathbf{A}^{\varepsilon}\right)$ a minimal solution of (2.2). Let

$$
\lambda_{\varepsilon}=\left\|\psi^{\varepsilon}\right\|_{L^{\infty}(\Omega)}, \quad \varphi^{\varepsilon}=\frac{\psi^{\varepsilon}}{\lambda_{\varepsilon}} .
$$

Recall that we have defined the tangential distance $d_{t}(x(s, t), \mathcal{N}(\partial \Omega))$ and have introduced the notations $\Omega_{\delta}$ and $\mathcal{N}(\partial \Omega)$ (see Section 1).

THEOREM 6.1. - Let

$$
\rho=\frac{\beta_{0}^{3 / 2}}{C_{1}}\left[H_{C_{3}}(\kappa)-\sigma\right]
$$


(1) Assume that $0<\rho \leqslant L_{1} \kappa^{-a}$, where $0<a \leqslant 1 / 3$ and $L_{1}$ is a positive constant. Then there exist positive constants $\alpha_{1}, c_{1}, l_{1}, \varepsilon_{1}$ and $M_{1}$ such that, for all $0<\varepsilon<\varepsilon_{1}$, we have

$$
\int_{\Omega}\left|\psi^{\varepsilon}\right|^{2} \exp \left(\frac{\alpha_{1}}{\sqrt{\varepsilon}}\left[\kappa_{\max }-\kappa_{r}(x)-l_{1} \varepsilon^{a}\right]\right) \mathrm{d} x \leqslant M_{1} \int_{\Omega_{c_{1} \varepsilon}}\left|\psi^{\varepsilon}\right|^{2} \mathrm{~d} x .
$$

(2) Assume that $L_{2} \kappa^{-1 / 3} \leqslant \rho=\mathrm{o}(1)$, where $L_{2}$ is a positive constant. Then there exist positive constants $\alpha_{2}, c_{2}, l_{2}, \varepsilon_{2}$ and $M_{2}$ such that, for all $0<\varepsilon<\varepsilon_{2}$, we have

$$
\int_{\Omega}\left|\psi^{\varepsilon}\right|^{2} \exp \left(\frac{\alpha_{2}}{\sqrt{\varepsilon}}\left[\kappa_{\max }-\kappa_{r}(x)-l_{2} \rho\right]\right) \mathrm{d} x \leqslant M_{2} \int_{\Omega_{c_{2} \varepsilon}}\left|\psi^{\varepsilon}\right|^{2} \mathrm{~d} x .
$$

(3) Assume that $\Omega$ is not a ball, and let $\varepsilon_{0}<\rho<\kappa_{\max }-\kappa_{\min }$. Then, there exist positive constants $\alpha_{3}, c_{3}, M_{3}$ and $\varepsilon_{3}$ such that, for all $0<\varepsilon<\varepsilon_{3}$, we have

$$
\int_{\Omega}\left|\psi^{\varepsilon}\right|^{2} \exp \left(\frac{\alpha_{3}}{\sqrt{\varepsilon}}\left[\kappa_{\max }-\kappa_{r}(s)-\rho\right]_{+}^{3 / 2}\right) \mathrm{d} x \leqslant \frac{M_{3}}{\varepsilon^{1 / 3}} \int_{\Omega_{c_{3} \varepsilon}}\left|\psi^{\varepsilon}\right|^{2} \mathrm{~d} x .
$$

In the proof of Theorem 6.1 we need the following notations. For $0<\delta<\delta_{0}, \mu>0$, we define

$$
\begin{aligned}
& \Gamma(\delta, \mu)=\left\{x \in \bar{\Omega}: \operatorname{dist}(x, \partial \Omega)<\delta, \kappa_{r}(x)>\kappa_{\max }-\mu\right\}, \\
& \gamma(z)=\sup \left\{\operatorname{dist}(x(s), \mathcal{N}(\partial \Omega)): x(s) \in \partial \Omega, \kappa_{r}(s) \geqslant \kappa_{\max }-z\right\}, \quad \text { for } z>0 .
\end{aligned}
$$

If $\Omega$ is not a ball and if $\kappa_{\min } \leqslant \beta \leqslant \kappa_{\max }$, we define

$$
\Gamma_{\beta}(\delta, \mu)=\left\{x \in \bar{\Omega}: \operatorname{dist}(x, \partial \Omega)<\delta, \kappa_{r}(x)>\beta-\mu\right\} .
$$

LEMMA 6.2. - Assume that $\Omega$ is a bounded domain of class $C^{4}$ in $\mathbb{R}^{2}$ and $\phi_{0}(s)=$ $\kappa_{\max }-\kappa_{r}(s)$. Then we have the following conclusions.

(1) There exists a positive constant $C$ such that $\left|\phi_{0}^{\prime}(s)\right|^{2} \leqslant C \phi_{0}(s)$.

(2) Assume that $\mathcal{N}(\partial \Omega)$ consists of non-degenerate maximum points of the curvature.

Then there exists a constant $\gamma_{0}>0$ such that $\gamma(z) \leqslant \gamma_{0} \sqrt{z}$ for $0<z<z^{*}$.

Proof. - Conclusion (1) is a standard result. We prove conclusion (2). Assume that $\mathcal{N}(\partial \Omega)$ consists of non-degenerate maximum points $x\left(s_{1}\right), \ldots, x\left(s_{n}\right)$ of the curvature. Then we can write

$$
\phi_{0}(s)=\rho_{0}(s)\left(s-s_{1}\right)^{2} \cdots\left(s-s_{n}\right)^{2},
$$

where $\rho_{0}(s)$ is a positive continuous function. Hence, we can find a positive constant $c>0$ such that $\phi_{0}(s) \geqslant c d_{t}(x(s), \mathcal{N}(\partial \Omega))^{2}$. So the conclusion is true.

Proof of Theorem 6.1. - Step 1. If $0<H_{C_{3}}(\kappa)-\sigma<C$ for large $\kappa$, then the number $t(\varepsilon)$ given in (2.13) satisfies

$$
\frac{t(\varepsilon)}{\varepsilon}=\beta_{0}^{3 / 2}\left[H_{C_{3}}(\kappa)-\sigma\right]+\mathrm{O}\left(\varepsilon^{1 / 3}\right), \text { for small } \varepsilon .
$$


This follows from (1.5) and the estimate

$$
\mu\left(\frac{1}{\varepsilon^{2}} \mathbf{F}\right)=\frac{1}{\varepsilon^{2}}\left[\beta_{0}-C_{1} \kappa_{\max } \varepsilon+\mathrm{O}\left(\varepsilon^{4 / 3}\right)\right]
$$

obtained in [15] (Theorem 11.1). In fact, since $\sigma=H_{C_{3}}(\kappa)+\mathrm{O}(1)=\frac{\kappa}{\beta_{0}}+\mathrm{O}(1)$ and $\varepsilon=1 / \sqrt{\sigma \kappa}$, we have $\varepsilon \sigma=\frac{1}{\sqrt{\beta_{0}}}+\mathrm{O}(\varepsilon)$. Using (6.5) and (1.5) we get

$$
\begin{aligned}
\frac{t(\varepsilon)}{\varepsilon} & =\frac{1}{\varepsilon}\left[\varepsilon^{2} \kappa^{2}-\varepsilon^{2} \mu\left(\frac{1}{\varepsilon^{2}} \mathbf{F}\right)\right]=\frac{\beta_{0}}{\varepsilon \sigma}\left[\frac{\kappa}{\beta_{0}}+\frac{C_{1}}{\beta_{0}} \kappa_{\max } \varepsilon \sigma-\sigma\right]+\mathrm{O}\left(\varepsilon^{1 / 3}\right) \\
& =\frac{\beta_{0}}{\varepsilon \sigma}\left[\frac{\kappa}{\beta_{0}}+\frac{C_{1}}{\beta_{0}^{3 / 2}} \kappa_{\max }-\sigma\right]+\mathrm{O}\left(\varepsilon^{1 / 3}\right)=\beta_{0}^{3 / 2}\left[H_{C_{3}}(\kappa)-\sigma\right]+\mathrm{O}\left(\varepsilon^{1 / 3}\right) .
\end{aligned}
$$

Step 2 . We prove an integral inequality.

Let $\chi$ be a $C^{1}$ function. As in (3.8) we have

$$
\begin{aligned}
Q_{\varepsilon^{2}, \mathbf{A}^{\varepsilon}}\left[\chi \varphi^{\varepsilon}\right] & \equiv \int_{\Omega}\left|\nabla_{\frac{1}{\varepsilon^{2}} \mathbf{A}^{\varepsilon}}\left(\chi \varphi^{\varepsilon}\right)\right|^{2} \mathrm{~d} x \\
& =\Re \int_{\Omega}\left(-\nabla_{\frac{1}{\varepsilon^{2}} \mathbf{A}^{\varepsilon}}^{2} \varphi^{\varepsilon}\right) \overline{\left(\chi^{2} \varphi^{\varepsilon}\right)} \mathrm{d} x+\int_{\Omega}\left|\varphi^{\varepsilon} \nabla \chi\right|^{2} \mathrm{~d} x \\
& =\kappa^{2} \int_{\Omega}\left(1-\lambda_{\varepsilon}^{2}\left|\varphi^{\varepsilon}\right|^{2}\right)\left|\chi \varphi^{\varepsilon}\right|^{2} \mathrm{~d} x+\int_{\Omega}\left|\varphi^{\varepsilon} \nabla \chi\right|^{2} \mathrm{~d} x .
\end{aligned}
$$

Using this and Proposition 3.7 we get

$$
\int_{\Omega} W_{\varepsilon}(x) \chi^{2}\left|\varphi^{\varepsilon}\right|^{2} \mathrm{~d} x \leqslant \int_{\Omega}\left\{\varepsilon^{2} \kappa^{2}\left(1-\lambda_{\varepsilon}^{2}\left|\varphi^{\varepsilon}\right|^{2}\right)\left|\chi \varphi^{\varepsilon}\right|^{2}+\varepsilon^{2}\left|\varphi^{\varepsilon} \nabla \chi\right|^{2}\right\} \mathrm{d} x .
$$

Let $\chi=\exp \left(\frac{\alpha \phi}{\sqrt{\varepsilon}}\right)$, where $\alpha$ and $\phi$ are to be chosen later. We get

$$
\int_{\Omega}\left|\varphi^{\varepsilon}\right|^{2} \exp \left(\frac{2 \alpha \phi}{\sqrt{\varepsilon}}\right)\left\{W_{\varepsilon}(x)-\varepsilon^{2} \kappa^{2}+\lambda_{\varepsilon}^{2} \varepsilon^{2} \kappa^{2}\left|\varphi^{\varepsilon}\right|^{2}-\alpha^{2} \varepsilon|\nabla \phi|^{2}\right\} \mathrm{d} x \leqslant 0 .
$$

The term involving $\left|\varphi^{\varepsilon}\right|^{4}$ in the left hand side of the above inequality has a positive sign, and will be neglected in the following (so the nonlinear effect is neglected, although this term could be useful to estimate the order of $\lambda_{\varepsilon}$ as $\varepsilon \rightarrow 0$ ).

In terms of the number $t(\varepsilon)$ defined in (2.13), we derive from (6.6) the following inequality:

$$
\int_{\Omega}\left|\varphi^{\varepsilon}\right|^{2} \exp \left(\frac{2 \alpha \phi}{\sqrt{\varepsilon}}\right)\left\{\left[W_{\varepsilon}(x)-\varepsilon^{2} \mu\left(\frac{1}{\varepsilon^{2}} \mathbf{F}\right)\right]-t(\varepsilon)-\alpha^{2} \varepsilon|\nabla \phi|^{2}\right\} \mathrm{d} x \leqslant 0 .
$$


Now, in $\Omega_{2 \varepsilon^{1 / 3}}$, using the upper bound (2.9) of $\mu\left(\frac{1}{\varepsilon^{2}} \mathbf{F}\right)$, the lower bound (3.25) of $W_{\varepsilon}(x)$, we have

$$
W_{\varepsilon}(x)-\mu\left(\frac{1}{\varepsilon^{2}} \mathbf{F}\right) \geqslant C_{1} \varepsilon\left[\kappa_{\max }-\kappa_{r}(x)\right]-C^{\prime} \varepsilon^{4 / 3},
$$

where $C^{\prime}$ is a positive constant independent of $\varepsilon$. In $\Omega \backslash \Omega_{2 \varepsilon^{1 / 3}}$ we have, for some constant $m>\left(1-\beta_{0}\right) / 2$,

$$
W_{\varepsilon}(x)-\varepsilon^{2} \mu\left(\frac{1}{\varepsilon^{2}} \mathbf{F}\right)-t(\varepsilon)-\alpha^{2} \varepsilon|\nabla \phi|^{2} \geqslant 1-\beta_{0}+\mathrm{o}(1) \geqslant m .
$$

Therefore, we have

$$
\begin{aligned}
& \int_{\Omega_{2 \varepsilon^{1 / 3}}}\left|\varphi^{\varepsilon}\right|^{2} \exp \left(\frac{2 \alpha \phi}{\sqrt{\varepsilon}}\right)\left\{C_{1}\left[\kappa_{\max }-\kappa_{r}(x)\right]-\frac{t(\varepsilon)}{\varepsilon}-C^{\prime} \varepsilon^{1 / 3}-\alpha^{2}|\nabla \phi|^{2}\right\} \mathrm{d} x \\
& \quad+\frac{m}{\varepsilon} \int_{\left\{\text {dist }(x, \partial \Omega) \geqslant 2 \varepsilon^{1 / 3}\right\}}\left|\varphi^{\varepsilon}\right|^{2} \exp \left(\frac{2 \alpha \phi}{\sqrt{\varepsilon}}\right) \mathrm{d} x \leqslant 0 .
\end{aligned}
$$

Step 3. We consider case (1).

Set $\phi(x)=\phi_{0}(x)=\kappa_{\max }-\kappa_{r}(x)$. Using conclusion (1) of Lemma 6.2, we can show that $\left|\nabla \phi_{0}(x)\right|^{2} \leqslant C \phi_{0}(x)$. Choose $\alpha$ such that $0<\alpha \leqslant \sqrt{\frac{C_{1}}{2 C}}$. From (6.7) we have

$$
\begin{aligned}
& \int_{\Omega_{2 \varepsilon^{1 / 3}}}\left|\varphi^{\varepsilon}\right|^{2} \exp \left(\frac{2 \alpha}{\sqrt{\varepsilon}} \phi_{0}(x)\right)\left\{\frac{C_{1}}{2} \phi_{0}(x)-\frac{t(\varepsilon)}{\varepsilon}-C^{\prime} \varepsilon^{1 / 3}\right\} \mathrm{d} x \\
& +\frac{m}{\varepsilon} \int_{\left\{\operatorname{dist}(x, \partial \Omega) \geqslant 2 \varepsilon^{1 / 3}\right\}}\left|\varphi^{\varepsilon}\right|^{2} \exp \left(\frac{2 \alpha}{\sqrt{\varepsilon}} \phi_{0}(x)\right) \mathrm{d} x \leqslant 0 .
\end{aligned}
$$

Suppose that $0<\rho \leqslant L_{1}^{\prime} \varepsilon^{1 / 3}$. Using (6.4) we find $t(\varepsilon)=\mathrm{O}\left(\varepsilon^{1+a}\right)$. From this and (6.8) we have

$$
\begin{gathered}
\int_{\Omega_{2 \varepsilon} \varepsilon^{1 / 3}}\left|\varphi^{\varepsilon}\right|^{2} \exp \left(\frac{2 \alpha}{\sqrt{\varepsilon}} \phi_{0}(x)\right)\left\{\frac{C_{1}}{2} \phi_{0}(x)-C^{*} \varepsilon^{a}\right\} \mathrm{d} x \\
+\frac{m}{\varepsilon} \int_{\left\{\operatorname{dist}(x, \partial \Omega) \geqslant 2 \varepsilon^{1 / 3}\right\}}\left|\varphi^{\varepsilon}\right|^{2} \exp \left(\frac{2 \alpha}{\sqrt{\varepsilon}} \phi_{0}(x)\right) \mathrm{d} x \leqslant 0,
\end{gathered}
$$

where $C^{*}$ is a constant independent of $\varepsilon$.

In the following, we choose $l_{1} \geqslant 4 C^{*} / C_{1}$. Note that, if $x=x(s, t) \in \Omega_{2 \varepsilon^{1 / 3}}$ । $\Gamma\left(2 \varepsilon^{1 / 3}, l_{1} \varepsilon^{a}\right)$, then $\phi_{0}(x)=\phi_{0}(x(s, t))=\phi_{0}(s) \geqslant l_{1} \varepsilon^{a} \geqslant \frac{4 C^{*}}{C_{1}} \varepsilon^{a}$. Therefore we have

$$
\begin{aligned}
& \int_{\Omega_{2 \varepsilon^{1 / 3} \backslash \Gamma\left(2 \varepsilon^{1 / 3}, l_{1} \varepsilon^{a}\right)}}\left|\varphi^{\varepsilon}\right|^{2} \exp \left(\frac{2 \alpha}{\sqrt{\varepsilon}} \phi_{0}(x)\right)\left\{\frac{C_{1}}{2} \phi_{0}(x)-C^{*} \varepsilon^{a}\right\} \mathrm{d} x \\
& \geqslant C^{*} \varepsilon^{a} \int_{\Omega_{2 \varepsilon^{1 / 3} \backslash \Gamma\left(2 \varepsilon^{1 / 3}, l_{1} \varepsilon^{a}\right)}}\left|\varphi^{\varepsilon}\right|^{2} \exp \left(\frac{2 \alpha}{\sqrt{\varepsilon}} \phi_{0}(x)\right) \mathrm{d} x .
\end{aligned}
$$


From this and (6.9) we get

$$
\begin{aligned}
& \int_{\Omega_{2 \varepsilon^{1 / 3} \backslash \Gamma\left(2 \varepsilon^{1 / 3}, l_{1} \varepsilon^{a}\right)}}\left|\varphi^{\varepsilon}\right|^{2} \exp \left(\frac{2 \alpha}{\sqrt{\varepsilon}} \phi_{0}(x)\right) \mathrm{d} x \\
& +\frac{m}{C^{*} \varepsilon^{1+a}} \int_{\left\{\operatorname{dist}(x, \partial \Omega) \geqslant 2 \varepsilon^{1 / 3}\right\}}\left|\varphi^{\varepsilon}\right|^{2} \exp \left(\frac{2 \alpha}{\sqrt{\varepsilon}} \phi_{0}(x)\right) \mathrm{d} x \\
& \leqslant \int_{\Gamma\left(2 \varepsilon^{1 / 3}, l_{1} \varepsilon^{a}\right)}\left|\varphi^{\varepsilon}\right|^{2} \exp \left(\frac{2 \alpha}{\sqrt{\varepsilon}} \phi_{0}(x)\right) \mathrm{d} x .
\end{aligned}
$$

Hence, for all $\varepsilon>0$ small, we have

$$
\int_{\Omega \backslash \Gamma\left(2 \varepsilon^{1 / 3}, l_{1} \varepsilon^{a}\right)}\left|\varphi^{\varepsilon}\right|^{2} \exp \left(\frac{2 \alpha}{\sqrt{\varepsilon}} \phi_{0}(x)\right) \mathrm{d} x \leqslant \int_{\Gamma\left(2 \varepsilon^{1 / 3}, l_{1} \varepsilon^{a}\right)}\left|\varphi^{\varepsilon}\right|^{2} \exp \left(\frac{2 \alpha}{\sqrt{\varepsilon}} \phi_{0}(x)\right) \mathrm{d} x .
$$

Using this and Lemma 4.5 we get

$$
\begin{aligned}
\int_{\Omega}\left|\varphi^{\varepsilon}\right|^{2} \exp \left(\frac{2 \alpha}{\sqrt{\varepsilon}} \phi_{0}(x)\right) \mathrm{d} x & \leqslant 2 \int_{\Gamma\left(2 \varepsilon^{1 / 3}, l_{1} \varepsilon^{a}\right)}\left|\varphi^{\varepsilon}\right|^{2} \exp \left(\frac{2 \alpha}{\sqrt{\varepsilon}} \phi_{0}(x)\right) \mathrm{d} x \\
& \leqslant 2 \exp \left(\frac{2 \alpha l_{1} \varepsilon^{a}}{\sqrt{\varepsilon}}\right) \int_{\Gamma\left(2 \varepsilon^{1 / 3}, l_{1} \varepsilon^{a}\right)}\left|\varphi^{\varepsilon}\right|^{2} \mathrm{~d} x \\
& \leqslant 2 M \exp \left(\frac{2 \alpha l_{1} \varepsilon^{a}}{\sqrt{\varepsilon}}\right) \int_{\Omega_{c \varepsilon}}\left|\varphi^{\varepsilon}\right|^{2} \mathrm{~d} x .
\end{aligned}
$$

From this we get (6.1) with $\alpha_{1}=2 \alpha, c_{1}=c$ and $M_{1}=2 M$.

Step 4. We consider case (2). Assume $L_{2}^{\prime} \varepsilon^{1 / 3} \ll \rho(\varepsilon)=o(1)$ as $\varepsilon \rightarrow 0$. From (6.4) we have

$$
a_{1} \varepsilon^{1 / 3} \ll \frac{t(\varepsilon)}{\varepsilon}=C_{1} \rho+\mathrm{O}\left(\varepsilon^{1 / 3}\right)=\mathrm{o}(1) .
$$

Choose $\phi(x)=\phi_{0}(x)=\kappa_{\max }-\kappa_{r}(x)$ and $\alpha$ as in case 1. Then (6.8) also holds, and

$$
\frac{C_{1}}{2} \phi_{0}(x)-\frac{t(\varepsilon)}{\varepsilon}-C^{\prime} \varepsilon^{1 / 3}=\frac{C_{1}}{2} \phi_{0}(x)-C_{1} \rho-\mathrm{O}\left(\varepsilon^{1 / 3}\right) .
$$

Choose $l_{2} \geqslant 4$. If $x=x(s, t) \in \Omega_{2 \varepsilon^{1 / 3}} \backslash \Gamma\left(2 \varepsilon^{1 / 3}, l_{2} \rho\right)$, then $\phi_{0}(x) \geqslant l_{2} \rho$, and, for all $\varepsilon>0$ small,

$$
\frac{C_{1}}{2} \phi_{0}(x)-\frac{t(\varepsilon)}{\varepsilon}-C^{\prime} \varepsilon^{1 / 3} \geqslant\left(\frac{C_{1} l_{2}}{2}-C_{1}\right) \rho-\mathrm{O}\left(\varepsilon^{1 / 3}\right) \geqslant C_{1} \rho .
$$

So, for small $\varepsilon>0$, we have

$$
\int_{\Omega_{2 \varepsilon^{1 / 3} \backslash \Gamma\left(2 \varepsilon^{1 / 3}, l_{2} \rho\right)}}\left|\varphi^{\varepsilon}\right|^{2} \exp \left(\frac{2 \alpha}{\sqrt{\varepsilon}} \phi_{0}(x)\right) \mathrm{d} x
$$




$$
\begin{aligned}
& +\frac{m}{C_{1} \rho \varepsilon} \int_{\left\{\operatorname{dist}(x, \partial \Omega) \geqslant 2 \varepsilon^{1 / 3}\right\}}\left|\varphi^{\varepsilon}\right|^{2} \exp \left(\frac{2 \alpha}{\sqrt{\varepsilon}} \phi_{0}(x)\right) \mathrm{d} x \\
\leqslant & \int_{\Gamma\left(2 \varepsilon^{1 / 3}, l_{2} \rho\right)}\left|\varphi^{\varepsilon}\right|^{2} \exp \left(\frac{2 \alpha}{\sqrt{\varepsilon}} \phi_{0}(x)\right) \mathrm{d} x .
\end{aligned}
$$

Hence

$$
\int_{\Omega \backslash \Gamma\left(2 \varepsilon^{1 / 3}, l_{2} \rho\right)}\left|\varphi^{\varepsilon}\right|^{2} \exp \left(\frac{2 \alpha}{\sqrt{\varepsilon}} \phi_{0}(x)\right) \mathrm{d} x \leqslant \int_{\Gamma\left(2 \varepsilon^{1 / 3}, l_{2} \rho\right)}\left|\varphi^{\varepsilon}\right|^{2} \exp \left(\frac{2 \alpha}{\sqrt{\varepsilon}} \phi_{0}(x)\right) \mathrm{d} x .
$$

Thus

$$
\begin{aligned}
\int_{\Omega}\left|\varphi^{\varepsilon}\right|^{2} \exp \left(\frac{2 \alpha}{\sqrt{\varepsilon}} \phi_{0}(x)\right) \mathrm{d} x & \leqslant 2 \int_{\Gamma\left(2 \varepsilon^{1 / 3}, l_{2} \rho\right)}\left|\varphi^{\varepsilon}\right|^{2} \exp \left(\frac{2 \alpha}{\sqrt{\varepsilon}} \phi_{0}(x)\right) \mathrm{d} x \\
& \leqslant 2 \exp \left(\frac{2 \alpha l_{2} \rho}{\sqrt{\varepsilon}}\right) \int_{\Gamma\left(2 \varepsilon^{1 / 3}, l_{2} \rho\right)}\left|\varphi^{\varepsilon}\right|^{2} \mathrm{~d} x \\
& \leqslant 2 M \exp \left(\frac{2 \alpha l_{2} \rho}{\sqrt{\varepsilon}}\right) \int_{\Omega_{c \varepsilon}}\left|\varphi^{\varepsilon}\right|^{2} \mathrm{~d} x .
\end{aligned}
$$

From this inequality we get (6.2) with $\alpha_{2}=2 \alpha, c_{2}=c$ and $M_{2}=2 M$.

Step 5. We consider case (3). Assume that $\Omega$ is not a ball, and $\varepsilon_{0}<\rho<\kappa_{\max }-\kappa_{\min }$. We introduce $\beta=\kappa_{\max }-\rho$. Then $\kappa_{\min }<\beta<\kappa_{\max }$. From (6.4) we have $t(\varepsilon)=C_{1}\left(\kappa_{\max }-\right.$ $\beta) \varepsilon+\mathrm{O}\left(\varepsilon^{4 / 3}\right)$. Define

$$
\phi(x)=\left[\beta-\kappa_{r}(x)\right]_{+}^{3 / 2}= \begin{cases}{\left[\beta-\kappa_{r}(x)\right]^{3 / 2}} & \text { if } \kappa_{r}(x) \leqslant \beta \\ 0 & \text { if } \kappa_{t}(s)>\beta\end{cases}
$$

Choose $\alpha>0$ small such that $\frac{9}{4} \alpha^{2}\left\|\kappa_{r}^{\prime}\right\|_{L^{\infty}}^{2}<\frac{C_{1}}{2}$. For all $x \in \Omega_{2 \varepsilon^{1 / 3}}$ we have

$$
\begin{aligned}
C_{1} & {\left[\kappa_{\max }-\kappa_{r}(x)\right]-\frac{t(\varepsilon)}{\varepsilon}-\alpha^{2}|\nabla \phi|^{2} } \\
& =C_{1}\left[\kappa_{\max }-\kappa_{r}(x)\right]-C_{1}\left[\kappa_{\max }-\beta\right]-\frac{9}{4} \alpha^{2}\left|\kappa_{r}^{\prime}(s)\right|^{2}\left[\beta-\kappa_{r}(x)\right]_{+}+\mathrm{O}\left(\varepsilon^{1 / 3}\right) \\
& \geqslant C_{1}\left[\beta-\kappa_{r}(x)\right]-\frac{C_{1}}{2}\left[\beta-\kappa_{r}(x)\right]_{+}+\mathrm{O}\left(\varepsilon^{1 / 3}\right) .
\end{aligned}
$$

From this and (6.7) we have, for some constant $C^{\prime \prime}>0$

$$
\begin{aligned}
& \int_{\Omega_{2 \varepsilon^{1 / 3}}}\left|\varphi^{\varepsilon}\right|^{2} \exp \left(\frac{2 \alpha \phi}{\sqrt{\varepsilon}}\right)\left\{2\left[\beta-\kappa_{r}(x)\right]-\left[\beta-\kappa_{r}(x)\right]_{+}-C^{\prime \prime} \varepsilon^{1 / 3}\right\} \mathrm{d} x \\
& \quad+\frac{2 m}{C_{1} \varepsilon} \int_{\left\{\operatorname{dist}(x, \partial \Omega) \geqslant 2 \varepsilon^{1 / 3}\right\}}\left|\varphi^{\varepsilon}\right|^{2} \exp \left(\frac{2 \alpha \phi}{\sqrt{\varepsilon}}\right) \mathrm{d} x \leqslant 0 .
\end{aligned}
$$


Choose $l_{3} \geqslant 2 C^{\prime \prime}$. On the set $\Gamma_{\beta}\left(2 \varepsilon^{1 / 3}, l_{3} \varepsilon^{1 / 3}\right)$ we have $\left[\beta-\kappa_{r}(x)\right]_{+} \leqslant l_{3} \varepsilon^{1 / 3}$. We can find $M^{\prime}>0$ such that, for $\varepsilon>0$ small

$$
\begin{aligned}
\left(l_{3}-\right. & \left.C^{\prime \prime}\right) \varepsilon^{1 / 3} \int_{\Omega \backslash \Gamma_{\beta}\left(2 \varepsilon^{1 / 3}, l_{3} \varepsilon^{1 / 3}\right)}\left|\varphi^{\varepsilon}\right|^{2} \exp \left(\frac{2 \alpha}{\sqrt{\varepsilon}}\left[\beta-\kappa_{r}(x)\right]_{+}^{3 / 2}\right) \mathrm{d} x \\
\leqslant & \int_{\Gamma_{\beta}\left(2 \varepsilon^{1 / 3}, l_{3} \varepsilon^{1 / 3}\right)}\left|\varphi^{\varepsilon}\right|^{2} \exp \left(\frac{2 \alpha}{\sqrt{\varepsilon}}\left[\beta-\kappa_{r}(x)\right]_{+}^{3 / 2}\right) \\
& \times\left\{\left[\beta-\kappa_{r}(x)\right]_{+}-2\left[\beta-\kappa_{r}(x)\right]+C^{\prime \prime} \varepsilon^{1 / 3}\right\} \mathrm{d} x \\
\leqslant & M^{\prime} \int_{\Gamma_{\beta}\left(2 \varepsilon^{1 / 3}, l_{3} \varepsilon^{1 / 3}\right)}\left|\varphi^{\varepsilon}\right|^{2} \mathrm{~d} x .
\end{aligned}
$$

Hence

$$
\begin{aligned}
& \int_{\Omega \backslash \Gamma_{\beta}\left(2 \varepsilon^{1 / 3}, l_{3} \varepsilon^{1 / 3}\right)}\left|\varphi^{\varepsilon}\right|^{2} \exp \left(\frac{2 \alpha}{\sqrt{\varepsilon}}\left[\beta-\kappa_{r}(x)\right]_{+}^{3 / 2}\right) \mathrm{d} x \\
& \leqslant \frac{M^{\prime}}{\left(l_{3}-C^{\prime \prime}\right) \varepsilon^{1 / 3}} \int_{\Gamma_{\beta}\left(2 \varepsilon^{1 / 3}, l_{3} \varepsilon^{1 / 3}\right)}\left|\varphi^{\varepsilon}\right|^{2} \mathrm{~d} x .
\end{aligned}
$$

Let $M^{\prime \prime}=M^{\prime} /\left(l_{3}-C^{\prime \prime}\right)+1$. For $\varepsilon>0$ small, we use the above inequality and Lemma 4.5 to get

$$
\begin{aligned}
\int_{\Omega}\left|\varphi^{\varepsilon}\right|^{2} \exp \left(\frac{2 \alpha}{\sqrt{\varepsilon}}\left[\beta-\kappa_{r}(x)\right]_{+}^{3 / 2}\right) \mathrm{d} x & \leqslant \frac{M^{\prime \prime}}{\varepsilon^{1 / 3}} \int_{\Gamma_{\beta}\left(2 \varepsilon^{1 / 3}, l_{3} \varepsilon^{1 / 3}\right)}\left|\varphi^{\varepsilon}\right|^{2} \mathrm{~d} x \\
& \leqslant \frac{M M^{\prime \prime}}{\varepsilon^{1 / 3}} \int_{\Omega_{c \varepsilon}}\left|\varphi^{\varepsilon}\right|^{2} \mathrm{~d} x .
\end{aligned}
$$

Hence (6.3) holds with $\alpha_{3}=2 \alpha, c_{3}=c$ and $M_{3}=M M^{\prime \prime}$.

Now Theorem 6.1 is proved.

Proof of Theorem 1.2. - Theorem 1.2 is a direct consequence of Theorem 6.1.

Proof of Theorem 1.3. - We prove conclusion (1). We keep the notations in Theorem 6.1.

Assume $0<H_{C_{3}}(\kappa)-\sigma \leqslant L_{1} \kappa^{-1 / 3}$. From Theorem 6.1 we see that, there exists $\varepsilon_{0}>0$ such that (6.1) holds with $a=1 / 3$ for all $0<\varepsilon<\varepsilon_{0}$, namely,

$$
\int_{\Omega}\left|\psi^{\varepsilon}\right|^{2} \exp \left(\frac{\alpha_{1}}{\sqrt{\varepsilon}}\left[\kappa_{\max }-\kappa_{r}(x)\right]\right) \mathrm{d} x \leqslant M_{1} \exp \left(\frac{\alpha_{1} l_{1}}{\varepsilon^{1 / 6}}\right) \int_{\Omega c_{1} \varepsilon}\left|\psi^{\varepsilon}\right|^{2} \mathrm{~d} x .
$$

Hence

$$
\int_{\Omega_{\delta_{0}} \backslash \Gamma\left(\delta_{0}, 2 l_{1} \varepsilon^{1 / 3}\right)}\left|\psi^{\varepsilon}\right|^{2} \mathrm{~d} x \leqslant M_{1} \exp \left(-\frac{\alpha_{1} l_{1}}{\varepsilon^{1 / 6}}\right) \int_{\Omega_{c_{1} \varepsilon}}\left|\psi^{\varepsilon}\right|^{2} \mathrm{~d} x .
$$


By the assumption, $\mathcal{N}(\partial \Omega)$ consists of non-degenerate maximum points of the curvature. From Lemma 6.2, conclusion (2), we have $\gamma(z) \leqslant \gamma_{0} \sqrt{z}$ for all $0<z<z^{*}$. So $\gamma\left(2 l_{1} \varepsilon^{1 / 3}\right) \leqslant \gamma_{0} \sqrt{2 l_{1}} \varepsilon^{1 / 6}$. Let $\ell=\gamma_{0} \sqrt{2 l_{1}}$. If $d_{t}(x, \mathcal{N}(\partial \Omega)) \geqslant \ell \varepsilon^{1 / 6}$ then $\kappa_{\max }-$ $\kappa_{r}(s(x)) \geqslant 2 l_{1} \varepsilon^{1 / 3}$. From (6.10) we get

$$
\int_{\left\{x \in \bar{\Omega}: \operatorname{dist}(x, \partial \Omega)<\delta_{0}, d_{t}(x, \mathcal{N}(\partial \Omega)) \geqslant \ell \varepsilon^{1 / 6}\right\}}\left|\psi^{\varepsilon}\right|^{2} \mathrm{~d} x \leqslant M_{1} \exp \left(-\frac{\alpha_{1} l_{1}}{\varepsilon^{1 / 6}}\right)\left\|\psi^{\varepsilon}\right\|_{L^{2}(\Omega)}^{2} .
$$

Recall that (3.4) holds for $\psi^{\varepsilon}$. So we have, for some positive constants $\alpha$ and $C$,

$$
\int_{\left\{r \varepsilon^{5 / 6} \leqslant \operatorname{dist}(x, \partial \Omega) \leqslant \delta_{0}\right\}}\left|\psi^{\varepsilon}\right|^{2} \mathrm{~d} x \leqslant C \exp \left(-\frac{\alpha r}{\varepsilon^{1 / 6}}\right) \int_{\Omega}\left|\psi^{\varepsilon}\right|^{2} \mathrm{~d} x .
$$

Choose $r=\alpha_{1} l_{1} / \alpha$. From (6.11) and (6.12) we have, for all small $\varepsilon$

$$
\int_{\bar{\Omega} \backslash\left\{x \in \bar{\Omega}: \operatorname{dist}(x, \partial \Omega)<r \varepsilon^{5 / 6}, d_{t}(x, \mathcal{N}(\partial \Omega))<\ell \varepsilon^{1 / 6}\right\}}\left|\psi^{\varepsilon}\right|^{2} \mathrm{~d} x \leqslant B_{1} \exp \left(-\frac{\alpha_{1} l_{1}}{\varepsilon^{1 / 6}}\right)\left\|\psi^{\varepsilon}\right\|_{L^{2}(\Omega)}^{2},
$$

where $B_{1}=M_{1}+C$. Choose positive constants $\ell_{1}, r_{1}, a_{1}$ and $\varepsilon_{1}^{\prime}$ such that, for $0<\varepsilon<\varepsilon_{1}^{\prime}$,

$$
\ell \varepsilon^{1 / 6} \leqslant \ell_{1} \kappa^{-1 / 6}, \quad r \varepsilon^{5 / 6} \leqslant r_{1} \kappa^{-5 / 6}, \quad \frac{\alpha_{1} l_{1}}{\varepsilon^{1 / 6}} \geqslant a_{1} \kappa^{1 / 6} .
$$

If $\kappa$ is large (so $\varepsilon$ is small), we have

$$
\int_{\bar{\Omega} \backslash\left\{x \in \bar{\Omega}: \operatorname{dist}(x, \partial \Omega)<r_{1} \kappa^{-5 / 6}, d_{t}(x, \mathcal{N}(\partial \Omega))<\ell_{1} \kappa^{-1 / 6}\right\}}\left|\psi^{\varepsilon}\right|^{2} \mathrm{~d} x \leqslant B_{1} \exp \left(-a_{1} \kappa^{1 / 6}\right)\left\|\psi^{\varepsilon}\right\|_{L^{2}(\Omega)}^{2} .
$$

So (1.8) is proved.

Conclusion (2) can be proved by a similar argument.

Remark 6.3. - It is interesting to estimate $\left\|\psi^{\varepsilon}\right\|_{L^{2}(\Omega)}^{4} /\left\|\psi^{\varepsilon}\right\|_{L^{4}(\Omega)}^{4}$ which, we believe, is proportional to $\left\|\psi^{\varepsilon}\right\|_{L^{2}(\Omega)}^{2}$ up to a higher order term, as $\varepsilon \rightarrow 0$. We may use (6.6) to obtain an estimate for $\left\|\psi^{\varepsilon}\right\|_{L^{\infty}(\Omega)}$ in terms of $\left\|\psi^{\varepsilon}\right\|_{L^{2}(\Omega)}^{4} /\left\|\psi^{\varepsilon}\right\|_{L^{4}(\Omega)}^{4}$.

\section{Acknowledgements}

This work was partially supported by the European union under the TMR Program EBRFXT 960001 (to Helffer) and by the National Natural Science Foundation of China, the Science Foundation of the Ministry of Education of China, the Zhejiang Provincial Natural Science Foundation of China, and NUS Academic Research Grant R-146-000022-112 (to Pan). 


\section{REFERENCES}

[1] S. Agmon, Lectures on Exponential Decay of Solutions of Second Order Elliptic Equations: Bounds on Eigenfunctions of $N$-Body Schrodinger Operators, Princeton University Press, 1982.

[2] P. Bauman, D. Phillips, Q. Tang, Stable nucleation for the Ginzburg-Landau system with an applied magnetic field, Arch. Rational Mech. Anal. 142 (1998) 1-43.

[3] A. Bernoff, P. Sternberg, Onset of superconductivity in decreasing fields for general domains, J. Math. Phys. 38 (1998) 1272-1284.

[4] C. Bolley, B. Helffer, An application of semi-classical analysis to the asymptotic study of the supercooling field of a superconducting material, Ann. Inst. Henri Poincaré, Physique Théorique 58 (1993) 189-233.

[5] S.J. Chapman, Nucleation of superconductivity in decreasing fields, European J. Appl. Math. 5 (1994), part 1, 449-468; part 2, 469-494.

[6] S.J. Chapman, S.D. Howison, J.R. Ockendon, Macroscopic models for superconductivity, SIAM Rev. 34 (1992) 529-560.

[7] M. Dauge, B. Helffer, Eigenvalues variation, I, Neumann problem for Sturm-Liouville operators, J. Differential Equations 104 (1993) 243-262.

[8] P.G. De Gennes, Superconductivity of Metals and Alloys, Benjamin, New York, 1966.

[9] M. del Pino, P. Felmer, P. Sternberg, Boundary concentration for eigenvalue problems related to the onset of superconductivity, Comm. Math. Phys. 210 (2000) 413-446.

[10] Q. Du, M. Gunzburger, J. Peterson, Analysis and approximation of the Ginzburg-Landau model of superconductivity, SIAM Rev. 34 (1992) 45-81.

[11] V. Ginzburg, L. Landau, On the theory of superconductivity, Soviet Phys. JETP 20 (1950) 1064-1082.

[12] T. Giorgi, D. Phillips, The breakdown of superconductivity due to strong fields for the Ginzburg-Landau model, SIAM J. Math. Anal. 30 (1999) 341-359.

[13] B. Helffer, Semi-Classical Analysis for the Schrödinger Operator and Applications, in: Lecture Notes in Mathematics, Vol. 1336, Springer-Verlag, 1988.

[14] B. Helffer, A. Mohamed, Semiclassical analysis for the ground state energy of a Schrödinger operator with magnetic wells, J. Funct. Anal. 138 (1996) 40-81.

[15] B. Helffer, A. Morame, Magnetic bottles in connection with superconductivity, J. Funct. Anal. 185 (2001) 604-680.

[16] K. Lu, X.-B. Pan, The first eigenvalue of Ginzburg-Landau operator, in: Bates, et al. (Eds.), Differential Equations and Applications, International Press, 1997, pp. 215-226.

[17] K. Lu, X.-B. Pan, Gauge invariant eigenvalue problems in $\mathbb{R}^{2}$ and in $\mathbb{R}_{+}^{2}$, Trans. Amer. Math. Soc. 352 (2000) 1247-1276.

[18] K. Lu, X.-B. Pan, Eigenvalue problems of Ginzburg-Landau operator in bounded domains, J. Math. Phys. 40 (1999) 2647-2670.

[19] K. Lu, X.-B. Pan, Estimates of the upper critical field for the Ginzburg-Landau equations of superconductivity, Phys. D 127 (1999) 73-104.

[20] K. Lu, X.-B. Pan, Surface nucleation of superconductivity in 3-dimension, J. Differential Equations 168 (2000) 386-452.

[21] K. Lu, X.-B. Pan, Surface nucleation of superconductivity, Methods and Applications of Analysis 8 (2001) 279-300.

[22] D. Saint-James, P. De Gennes, Onset of superconductivity in decreasing fields, Phys. Lett. 6 (5) (1963) 306-308.

[23] D. Saint-James, G. Sarma, E.J. Thomas, Type II Superconductivity, Pergamon Press, Oxford, 1969.

[24] M. Tinkham, Introduction to Superconductivity, McGraw-Hill, New York, 1975. 